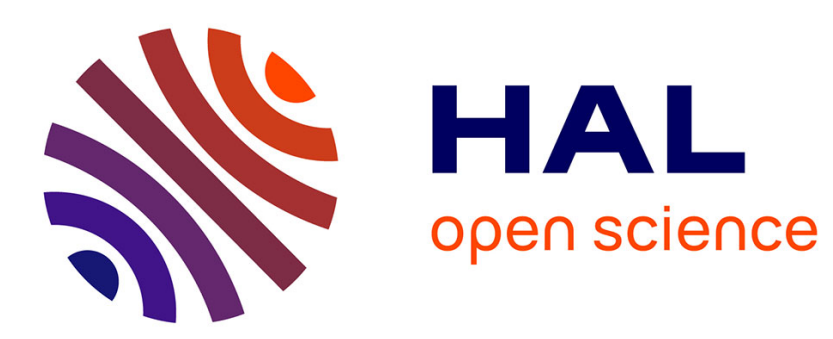

\title{
A differential geometric approach to nonlinear filtering: the projection filter
}

\author{
Damiano Brigo, Bernard Hanzon, François Le Gland
}

\section{To cite this version:}

Damiano Brigo, Bernard Hanzon, François Le Gland. A differential geometric approach to nonlinear filtering: the projection filter. [Research Report] 2598, INRIA Rennes - Bretagne Atlantique. 1995. hal-02101519

\section{HAL Id: hal-02101519 \\ https://hal.inria.fr/hal-02101519}

Submitted on 16 Apr 2019

HAL is a multi-disciplinary open access archive for the deposit and dissemination of scientific research documents, whether they are published or not. The documents may come from teaching and research institutions in France or abroad, or from public or private research centers.
L'archive ouverte pluridisciplinaire HAL, est destinée au dépôt et à la diffusion de documents scientifiques de niveau recherche, publiés ou non, émanant des établissements d'enseignement et de recherche français ou étrangers, des laboratoires publics ou privés. 


\section{A Differential Geometric Approach to Nonlinear Filtering : the Projection Filter}

Damiano Brigo Bernard Hanzon, Francois Le Gland

$\mathbf{N}^{\circ} 2598$

Juin 1995

PROGRAMME 5

\section{apport \\ de recherche}





\title{
RINRIA
}

\section{A Differential Geometric Approach to Nonlinear Filtering : the Projection Filter}

\author{
Damiano Brigo* Bernard Hanzon*, Francois Le Gland ** \\ Programme 5 - Traitement du signal, automatique et productique \\ Projet AS \\ Rapport de recherche $\mathrm{n}^{\circ} 2598$ - Juin 1995 - 50 pages
}

\begin{abstract}
This paper deals with a new and systematic method of approximating exact nonlinear filters with finite dimensional filters. The method used here is based on the differential geometric approach to statistics. The projection filter is derived in the case of exponential families. A characterization of the filters is given in terms of an assumed density principle. An a posteriori measure of the performance of the projection filter is defined. Applications to particular systems, and numerical schemes which can be used to implement the projection filter are given in the final part. The results of simulations for the cubic sensor are discussed.
\end{abstract}

Key-words: finite dimensional filtering, assumed density filter, projection filter, Fisher information metric, differential geometry and statistics.

(Résumé : tsvp)

This work was partially supported by the European Economic Community, under the SCIENCE project System Identification, project number SC1*-CT92-0779, and by the Army Research Office, under grant DAAH04-95-1-0164. Damiano Brigo was also supported by an Advanced Studying Fellowship of the University of Padua.

*Department of Econometrics, Free University Amsterdam, De Boelelaan 1105, 1081 HV Amsterdam, The Netherlands - \{dbrigo,bhanzon\}@econ.vu.nl

**IRISA / INRIA, Campus de Beaulieu, 35042 Rennes Cédex, France - legland@irisa.fr

Unité de recherche INRIA Rennes

IRISA, Campus universitaire de Beaulieu, 35042 RENNES Cedex (France)

Téléphone : (33) 99847100 - Télécopie : (33) 99847171 


\section{Une Approche du Filtrage Non-Linéaire Fondée sur la Géométrie Différentielle : le Filtre par Projection}

Résumé : Cet article propose une méthode nouvelle et systématique pour l'approximation d'un filtre non-linéaire exact par un filtre de dimension finie. La méthode repose sur l'utilisation d'outils de géométrie différentielle en statistique. L'équation du filtre par projection est établie dans le cas des familles exponentielles, et on en donne une caractérisation en tant que filtre de forme donnée. On définit également une mesure a posteriori de la qualité de l'approximation. Dans la dernière partie, on étudie quelques exemples, et on propose un schéma numérique pour la mise en œuvre du filtre par projection. Finalement, on présente des résultats de simulations pour le problème du senseur cubique.

Mots-clé : filtre de dimension finie, assumed density filter, projection filter, information de Fisher, géométrie différentielle et statistique 


\section{Introduction}

The filtering problem consists in estimating the state of a stochastic differential system from noisy observations. In the linear Gaussian case the problem was solved by Kalman, who introduced the well known Kalman filter, a finite dimensional system of equations for the first two conditional moments of the state given the observations. In the linear context this provides also the whole conditional density of the state given the observations, as this density is Gaussian and hence characterized by the first two moments. In the general nonlinear case, the filtering problem consists in computing the conditional density of the state given the observations. This density is the solution of a stochastic partial differential equation, the Kushner-Stratonovich equation. The general nonlinear problem is far more complicated because the resulting nonlinear filter is not finite dimensional in general. A well known approximation method is the extended Kalman filter (EKF): one linearizes around the current estimate obtaining a locally linear system, and then applies the Kalman filter equations. This procedure is usually justified on the basis of heuristic considerations, and not much is known about its efficiency, except in the case of small observation noise, see Picard [19], [17] and [18].

Another choice in the nonlinear case is the Gaussian assumed density filter (GADF), obtained by assuming the conditional density to be Gaussian, closing under this assumption the set of exact equations for the first two moments and producing a finite dimensional filter. This is dangerous, because assuming a false hypothesis one can deduce everything.

In 1987, Hanzon [6] introduced the projection filter (PF), which is a finite dimensional nonlinear filter based on the differential geometric approach to statistics. The projection filter is obtained by projecting the Kushner-Stratonovich equation onto the tangent space of a finite dimensional manifold of probability densities, according to the Fisher information metric and its extension to the infinite dimensional space of square roots of densities, the Hellinger distance.

Later on, in 1991, it was proved in Hanzon and Hut [8] that if one projects onto the tangent space of the finite dimensional manifold of Gaussian densities, the resulting PF coincides with an assumed density filter which is obtained as follows: one computes the first two conditional moments equations in McShane-Fisk-Stratonovich (MFS) form, and then assumes the conditional density to be Gaussian, closing in this way the equations for the first two moments. We call this filter MFS-based GADF. Its efficiency has been recently studied in Brigo [2], in the case of small observation noise. In [8] it was also proven that what we described above is in general not the same as assuming a Gaussian density in the Itô equations for the first two moments and then transforming the obtained filter in MFS form : the MFS-based GADF is not just an MFS version of the Itô-based GADF. The equivalence between the MFS-based GADF and the Gaussian PF is very important when generalized to exponential families, because it gives a simple characterization of the exponential projection filter (EPF) which is independent of geometric concepts. In fact we will see that in principle the EPF can be derived as an assumed density filter : one can just write the MFS equations for the $m$ conditional expectations of the exponent functions of the selected exponential family, and then assume the conditional density to be exponential and characterized by such

$\operatorname{RR}{ }^{\circ} 2598$ 
expectations, obtaining in this way a closed set of stochastic differential equation. We shall prove that this is the same as the EPF obtained by projecting the right-hand-side of the Kushner-Stratonovich equation on the selected exponential family.

The purpose of this paper is to provide an introduction to the projection filter. We provide a rigorous definition of the $\mathrm{PF}$ in the case of a manifold of exponential probability densities. We also present some formulae concerning auxiliary quantities, such as the projection residual (PR), the purpose of which is to provide a local measure of the quality of the filter behaviour. We develop explicit formulae for the particular example of the cubic sensor. The filters are derived by using the geometric approach, but in principle the reader can rederive them by using the assumed density idea without using any Riemannian geometry. Finally, we present some numerical simulations and comparisons for the cubic sensor, between the projection filter and the numerical solution of the nonlinear filtering equation.

\section{Statistical manifolds}

On the measurable space $\left(\mathbf{R}^{n}, \mathcal{B}\left(\mathbf{R}^{n}\right)\right)$ we consider a non-negative and $\sigma$-finite measure $\lambda$, and we define $\mathcal{M}(\lambda)$ to be the set of all non-negative and finite measures $\mu$ which are absolutely continuous w.r.t. $\lambda$, and whose density

$$
p_{\mu}=\frac{d \mu}{d \lambda}
$$

is positive $\lambda$-a.e. For simplicity, we restrict ourselves in this paper to the case where $\lambda$ is the Lebesgue measure on $\mathbf{R}^{n}$.

In the following, we denote by $H(\lambda)$ the set of all the densities of measures contained in $\mathcal{M}(\lambda)$. Notice that, as all the measures in $\mathcal{M}(\lambda)$ are non-negative and finite, we have that if $p$ is a density in $H(\lambda)$ then $p \in L_{1}(\lambda)$, that is $(\sqrt{p})^{2} \in L_{1}(\lambda)$ and then $\sqrt{p} \in L_{2}(\lambda)$. The above remark implies that the set $\mathcal{R}(\lambda):=\{\sqrt{p}: p \in H(\lambda)\}$ of square roots of densities of $H(\lambda)$ is a subset of $L_{2}(\lambda)$. Notice that all $\sqrt{p}$ in $\mathcal{R}(\lambda)$ satisfy $\sqrt{p(x)}>0$, for all $x \in \mathbf{R}^{n}$. The above remarks lead to the definition of the following metric in $\mathcal{R}(\lambda)$, see $[7]: d_{\mathcal{R}}\left(\sqrt{p_{1}}, \sqrt{p_{2}}\right):=$ $\left\|\sqrt{p_{1}}-\sqrt{p_{2}}\right\|$, where $\|\cdot\|$ denotes the norm of the Hilbert space $L_{2}(\lambda)$. This leads to the Hellinger metric on $H(\lambda)$ (or $\mathcal{M}(\lambda)$ ), obtained by using the bijection between densities (or measures) and square roots of densities : if $\mu_{1}$ and $\mu_{2}$ are the measures having densities $p_{1}$ and $p_{2}$ w.r.t. $\lambda$, the Hellinger metric is defined as $d_{\mathcal{M}}\left(\mu_{1}, \mu_{2}\right)=d_{H}\left(p_{1}, p_{2}\right)=d_{\mathcal{R}}\left(\sqrt{p_{1}}, \sqrt{p_{2}}\right)$. It can be shown, see e.g. [7], that the distance $d_{\mathcal{M}}\left(\mu_{1}, \mu_{2}\right)$ in $\mathcal{M}(\lambda)$ is defined independently of the particular $\lambda$ we choose as basic measure, as long as both $\mu_{1}$ and $\mu_{2}$ are absolutely continuous w.r.t. $\lambda$. As one can always find a $\lambda$ such that both $\mu_{1}$ and $\mu_{2}$ are absolutely continuous w.r.t. $\lambda$ (take for example $\lambda:=\left(\mu_{1}+\mu_{2}\right) / 2$ ), the distance is well defined on the set of all finite and positive measures on $(\Omega, \mathcal{F})$. Note that $\mathcal{R}(\lambda)$ is not a submanifold of $L_{2}(\lambda)$, in particular it is not open in $L_{2}(\lambda)$.

In the following we give a very quick review of the main concepts we need from differential geometry. For the basic definitions and a more technical introduction on manifolds, tangent vectors and related concepts we refer to the literature, see for example [1], the references 
given therein. Consider an open subset $M$ of $L_{2}(\lambda)$. Let $x$ be a point of $M$. Let $\gamma$ be a curve on $M$ around $x$, i.e. a differentiable map between an open neighborhood of $0 \in \mathbf{R}$ and $M$ such that $\gamma(0)=x$. We can define the tangent vector to $\gamma$ at $x$ as the Fréchet derivative $D \gamma(0)$. The derivative $D \gamma(0)$ is the linear map defined in $\mathbf{R}$ around 0 and taking values in $L_{2}(\lambda)$ such that the following limit holds:

$$
\lim _{|h| \rightarrow 0} \frac{\|\gamma(h)-\gamma(0)-D \gamma(0) \cdot h\|}{|h|}=0 .
$$

The map $D \gamma(0)$ approximates linearly the change of $\gamma$ around $x$. Let $\mathcal{C}_{x}(M)$ be the set of all the curves on $M$ around $x$. If we consider the space

$$
L_{x} M:=\left\{D \gamma(0): \gamma \in \mathcal{C}_{x}(M)\right\},
$$

of tangent vectors to all the possible curves on $M$ around $x$, we obtain again the space $L_{2}(\lambda)$. This is due to the fact that for every $v \in L_{2}(\lambda)$ we can always consider the straight line $\gamma^{v}(h):=x+h v$. Since $M$ is open, $\gamma^{v}(h)$ takes values in $M$ for $|h|$ small enough. Of course $D \gamma^{v}(0)=v$, so that indeed $L_{x} M=L_{2}(\lambda)$. The situation becomes different if we consider an $m$-dimensional manifold $N$ imbedded in $L_{2}(\lambda)$. We can consider the induced $L_{2}$ structure on $N$ as follows : suppose $x \in N$, and define again

$$
L_{x} N:=\left\{D \gamma(0): \gamma \in \mathcal{C}_{x}(N)\right\} .
$$

This is a linear subspace of $L_{2}(\lambda)$ called the tangent vector space at $x$, which does not coincide with $L_{2}(\lambda)$ in general (due to the finite dimension of $N$ ). The set of all tangent vectors at all points $x$ of $N$ is called the tangent bundle, and will be denoted by $L N$. In our work we shall consider finite dimensional manifolds $N$ embedded in $L_{2}(\lambda)$, which are contained in $\mathcal{R}(\lambda)$ as a set, i.e. $N \subset \mathcal{R}(\lambda) \subset L_{2}(\lambda)$, so that usually $x=\sqrt{p}$. It may be important to point out that, although we are using square roots of densities in order to keep the $L_{2}$ structure, once we have a finite dimensional manifold $N$, we can consider any of the embeddings $\sqrt{p} \mapsto \mu_{p}$, or $\sqrt{p} \mapsto p$, focusing on manifolds of probability measures $\mu_{p}$, or their densities $p$ rather than on their square roots $\sqrt{p}$.

If $N$ is $m$-dimensional, it is locally homeomorphic to $\mathbf{R}^{m}$, and it may be described locally by a chart : if $\sqrt{p} \in N$, there exists a pair $\left(S^{1 / 2}, \phi\right)$ with $S^{1 / 2}$ open neighbourhood of $\sqrt{p}$ in $N$ and $\phi: S^{1 / 2} \rightarrow \Theta$ homeomorphism of $S^{1 / 2}$ onto an open subset $\Theta$ of $\mathbf{R}^{m}$. By considering the inverse map $i$ of $\phi$,

$$
\begin{aligned}
i: \Theta & \longrightarrow S^{1 / 2} \\
\theta & \longmapsto \sqrt{p(\cdot, \theta)}
\end{aligned}
$$

we can express $S^{1 / 2}$ as

$$
i(\Theta)=\{\sqrt{p(\cdot, \theta)}, \theta \in \Theta\}=S^{1 / 2} .
$$

We shall denote by $S$ the following family of probability densities :

$$
S=\{p(\cdot, \theta): \theta \in \Theta\},
$$

RR $n^{\circ} 2598$ 
where $\Theta \subseteq \mathbf{R}^{m}$ and we will work only with the single coordinate chart $\left(S^{1 / 2}, \phi\right)$ as it is done in [1]. From the fact that $\left(S^{1 / 2}, \phi\right)$ is a chart, it follows that

$$
\left\{\frac{\partial i(\cdot, \theta)}{\partial \theta_{1}}, \cdots, \frac{\partial i(\cdot, \theta)}{\partial \theta_{m}}\right\}
$$

is a set of linearly independent vectors in $L_{2}(\lambda)$. In such a context, let us see what the vectors of $L \sqrt{p(\cdot, \theta)} S^{1 / 2}$ are. We can consider a curve in $S^{1 / 2}$ around $\sqrt{p(\cdot, \theta)}$ to be of the form $\gamma: h \mapsto \sqrt{p(\cdot, \theta(h))}$, where $h \mapsto \theta(h)$ is a curve in $\Theta$ around $\theta$. Then, according to the chain rule, we compute the following Fréchet derivative:

$$
D \gamma(0)=\left.D \sqrt{p(\cdot, \theta(h))}\right|_{h=0}=\sum_{k=1}^{m} \frac{\partial \sqrt{p(\cdot, \theta)}}{\partial \theta_{k}} \dot{\theta}_{k}(0)=\sum_{k=1}^{m} \frac{1}{2 \sqrt{p(\cdot, \theta)}} \frac{\partial p(\cdot, \theta)}{\partial \theta_{k}} \dot{\theta}_{k}(0) .
$$

We obtain that a basis for the tangent vector space at $\sqrt{p(\cdot, \theta)}$ to the space $S^{1 / 2}$ of square roots of densities of $S$ is given by :

$$
L_{\sqrt{p(\cdot, \theta)}} S^{1 / 2}=\operatorname{span}\left\{\frac{1}{2 \sqrt{p(\cdot, \theta)}} \frac{\partial p(\cdot, \theta)}{\partial \theta_{1}}, \cdots, \frac{1}{2 \sqrt{p(\cdot, \theta)}} \frac{\partial p(\cdot, \theta)}{\partial \theta_{m}}\right\} .
$$

As $i$ is the inverse of a chart, these vectors are actually linearly independent, and they indeed form a basis of the tangent vector space. One has to be careful, because if this were not true, the dimension of the above spanned space could drop. As an example, consider the curved exponential family

$$
S=\left\{p(x, \theta)=\exp \left[-\theta_{1}^{3} x-\left(\theta_{2}^{2}+1\right) x^{2}-\psi(\theta)\right], \theta \in \Theta \subset \mathbf{R}^{2}\right\}
$$

where $\psi$ is the normalizing constant. It is immediate to check that at $\left(\theta_{1}, \theta_{2}\right)=(0,0)$ - assuming this point is in $\Theta$ - the linear space defined in (1) above reduces to a one dimensional subspace of $L_{2}$. This happens because $\left(S^{1 / 2}, \phi\right)$ is not a chart for the manifold $N$ : it describes a different differential structure. The inner product of any two basis elements is defined, according to the $L_{2}$ inner product

$$
\begin{aligned}
\left\langle\frac{1}{2 \sqrt{p(\cdot, \theta)}} \frac{\partial p(\cdot, \theta)}{\partial \theta_{i}}, \frac{1}{2 \sqrt{p(\cdot, \theta)}} \frac{\partial p(\cdot, \theta)}{\partial \theta_{j}}\right\rangle & =\frac{1}{4} \int \frac{1}{p(x, \theta)} \frac{\partial p(x, \theta)}{\partial \theta_{i}} \frac{\partial p(x, \theta)}{\partial \theta_{j}} d \lambda(x) \\
& =\frac{1}{4} g_{i j}(\theta) .
\end{aligned}
$$

This is, up to the numeric factor $\frac{1}{4}$, the Fisher information metric, see [1], [6], [7], [8]. The matrix $g(\theta)=\left(g_{i j}(\theta)\right)$ is called the Fisher information matrix.

Next, we introduce the orthogonal projection between any linear subspace $V$ of $L_{2}(\lambda)$ containing the finite dimensional tangent vector space (1) and the tangent vector space (1) itself. Let us remember that our basis is not orthogonal, so that we have to project according 
to the following formula:

$$
\begin{aligned}
\Pi: H & \longrightarrow \operatorname{span}\left\{w_{1}, \ldots, w_{m}\right\} \\
v & \longmapsto \sum_{i=1}^{m}\left[\sum_{j=1}^{m} W^{i j}\left\langle v, w_{j}\right\rangle\right] w_{i}
\end{aligned}
$$

where $H$ is an Hilbert space, $\left\{w_{1}, \cdots, w_{m}\right\}$ are $m$ linearly independent vectors, $W:=$ $\left(\left\langle w_{i}, w_{j}\right\rangle\right)$ is the matrix formed by all the possible inner products of such linearly independent vectors, and $\left(W^{i j}\right)$ is the inverse of the matrix $W$. In our context $\left\{w_{1}, \cdots, w_{m}\right\}$ are the vectors in (1), and of course $W$ is, up to the numeric factor $\frac{1}{4}$, the Fisher information matrix given by (2) or (4). Then we obtain the following projection formula, where $\left(g^{i j}(\theta)\right)$ is the inverse of the Fisher information matrix $\left(g_{i j}(\theta)\right)$ :

$$
\begin{aligned}
\Pi_{\theta}: L_{2}(\lambda) \supseteq V & \longrightarrow \operatorname{span}\left\{\frac{1}{2 \sqrt{p(\cdot, \theta)}} \frac{\partial p(\cdot, \theta)}{\partial \theta_{1}}, \cdots, \frac{1}{2 \sqrt{p(\cdot, \theta)}} \frac{\partial p(\cdot, \theta)}{\partial \theta_{m}}\right\} \\
v & \longmapsto \sum_{i=1}^{m}\left[\sum_{j=1}^{m} 4 g^{i j}(\theta)\left\langle v, \frac{1}{2 \sqrt{p(\cdot, \theta)}} \frac{\partial p(\cdot, \theta)}{\partial \theta_{j}}\right\rangle\right] \frac{1}{2 \sqrt{p(\cdot, \theta)}} \frac{\partial p(\cdot, \theta)}{\partial \theta_{i}} .
\end{aligned}
$$

Let us go back to the definition of tangent vectors for our statistical manifold. Amari [1] uses a different representation of tangent vectors to $S$ at $p$. Without exploring all the assumptions needed, let us say that Amari defines an isomorphism between the actual tangent space and the vector space

$$
\operatorname{span}\left\{\frac{\partial \log p(\cdot, \theta)}{\partial \theta_{1}}, \cdots, \frac{\partial \log p(\cdot, \theta)}{\partial \theta_{m}}\right\} .
$$

On this representation of the tangent space, Amari defines a Riemannian metric given by

$$
E_{p(\cdot, \theta)}\left\{\frac{\partial \log p(\cdot, \theta)}{\partial \theta_{i}} \frac{\partial \log p(\cdot, \theta)}{\partial \theta_{j}}\right\},
$$

where $E_{p}\{\cdot\}$ denotes the expectation w.r.t. the probability density $p$. This is again the Fisher information metric, and indeed this is the most frequent definition of Fisher metric. In fact, it is easy to check that

$$
\begin{gathered}
E_{p(\cdot, \theta)}\left\{\frac{\partial \log p(\cdot, \theta)}{\partial \theta_{i}} \frac{\partial \log p(\cdot, \theta)}{\partial \theta_{j}}\right\}=\int \frac{\partial \log p(x, \theta)}{\partial \theta_{i}} \frac{\partial \log p(x, \theta)}{\partial \theta_{j}} p(x, \theta) d \lambda(x) \\
=\int \frac{1}{p(x, \theta)} \frac{\partial p(x, \theta)}{\partial \theta_{i}} \frac{\partial p(x, \theta)}{\partial \theta_{j}} d \lambda(x)=g_{i j}(\theta) .
\end{gathered}
$$

From the above relation and from (2) it is clear that, up to the numeric factor $\frac{1}{4}$, the Fisher information metric and the Hellinger metric coincide on the two representations of 
the tangent space to $S$ at $p(\cdot, \theta)$. There is another way of measuring how close two densities of $S$ are. Consider the Kullback-Leibler information between two densities $p$ and $q$ of $H(\lambda)$ :

$$
K(p, q):=\int \log \frac{p(x)}{q(x)} p(x) d \lambda(x)=E_{p}\left\{\log \frac{p}{q}\right\} .
$$

This is not a metric, since it is not symmetric and it does not satisfy the triangular inequality. When applied to a finite dimensional manifold such as $S$, both the Kullback-Leibler information and the Hellinger distance are particular cases of $\alpha$-divergence, see [1] for the details. One can show that the Fisher metric and the Kullback-Leibler information coincide infinitesimally. Indeed, consider the two densities $p(\cdot, \theta)$ and $p(\cdot, \theta+d \theta)$ of $S$. By expanding in Taylor series, we obtain

$$
\begin{aligned}
K(p(\cdot, \theta), p(\cdot, \theta+d \theta))= & -\sum_{i=1}^{m} E_{p(\cdot, \theta)}\left\{\frac{\partial \log p(\cdot, \theta)}{\partial \theta_{i}}\right\} d \theta_{i} \\
& -\sum_{i, j=1}^{m} E_{p(\cdot, \theta)}\left\{\frac{\partial^{2} \log p(\cdot, \theta)}{\partial \theta_{i} \partial \theta_{j}}\right\} d \theta_{i} d \theta_{j}+O\left(|d \theta|^{3}\right) \\
& =\sum_{i, j=1}^{m} g_{i j}(\theta) d \theta_{i} d \theta_{j}+O\left(|d \theta|^{3}\right) .
\end{aligned}
$$

We conclude this section with a lemma on exponential families, which will be used throughout the paper, see e.g. Amari [1]. We shall use the following equivalent notations for partial differentiation:

$$
\frac{\partial^{k}}{\partial \theta_{i_{1}} \cdots \partial \theta_{i_{k}}}=\partial_{i_{1}, \cdots, i_{k}}^{k} .
$$

Definition 2.1 Let $\left\{c_{1}, \cdots, c_{m}\right\}$ be linearly independent scalar functions defined on $\mathbf{R}^{n}$, and assume that the convex set

$$
\Theta_{0}:=\left\{\theta \in \mathbf{R}^{m}: \psi(\theta)=\log \int \exp \left[\theta^{T} c(x)\right] d \lambda(x)<\infty\right\},
$$

has non-empty interior. Then

$$
S=\{p(\cdot, \theta), \theta \in \Theta\}, \quad p(x, \theta):=\exp \left[\theta^{T} c(x)-\psi(\theta)\right],
$$

where $\Theta \subseteq \Theta_{0}$ is open, is called an exponential family of probability densities.

Remark 2.2 Given linearly independent scalar functions $\left\{c_{1}, \cdots, c_{m}\right\}$ defined on $\mathbf{R}^{n}$, it may happen that the densities $\exp \left[\theta^{T} c(x)\right]$ are not integrable. However, it is always possible to extend the family so as to deal with integrable densities only. Indeed, assume that there exist $K>0$ and $r \geq 0$ such that

$$
|c(x)| \leq K\left(1+|x|^{r}\right),
$$


for all $x \in \mathbf{R}^{n}$. Define $d(x):=|x|^{s}$ for all $x \in \mathbf{R}^{n}$, and some $s>r$. Then

$$
S^{\prime}:=\left\{p^{\prime}(\cdot, \theta, \mu), \theta \in \mathbf{R}^{m}, \mu>0\right\}, \quad p^{\prime}(x, \theta, \mu):=\exp \left[\theta^{T} c(x)-\mu d(x)-\psi^{\prime}(\theta, \mu)\right],
$$

is an exponential family of densities, with a non-empty open parameter set.

Lemma 2.3 Let

$$
S=\{p(\cdot, \theta), \theta \in \Theta\}, \quad p(x, \theta):=\exp \left[\theta^{T} c(x)-\psi(\theta)\right],
$$

where $\Theta \subset \mathbf{R}^{m}$ is open, be an exponential family of probability densities. Then the function $\psi$ is infinitely differentiable in $\Theta$

$$
\begin{gathered}
E_{p(\cdot, \theta)}\left\{c_{i}\right\}=\partial_{i} \psi(\theta)=: \eta_{i}(\theta), \\
E_{p(, \theta)}\left\{c_{i} c_{j}\right\}=\partial_{i j}^{2} \psi(\theta)+\partial_{i} \psi(\theta) \partial_{j} \psi(\theta),
\end{gathered}
$$

and more generally

$$
E_{p(, \theta)}\left\{c_{i_{1}} \cdots c_{i_{k}}\right\}=\exp [-\psi(\theta)] \frac{\partial^{k}}{\partial \theta_{i_{1}} \cdots \partial \theta_{i_{k}}} \exp [\psi(\theta)] .
$$

The Fisher information matrix satisfies

$$
g_{i j}(\theta)=\partial_{i j}^{2} \psi(\theta)=\partial_{i} \eta_{j}(\theta) .
$$

In the particular case where

$$
c_{i}(x)=x^{i}, \quad i=1, \cdots, m
$$

the following recursion formula holds, with $\eta_{0}(\theta):=1$ : for any nonnegative integer $i$

$$
\eta_{m+i}(\theta):=E_{p(\cdot, \theta)}\left\{x^{m+i}\right\}=-\frac{1}{m \theta_{m}}\left[\begin{array}{lllll}
(i+1) \theta_{1} & 2 \theta_{2} & \cdots & (m-1) \theta_{m-1}
\end{array}\right]\left[\begin{array}{c}
\eta_{i}(\theta) \\
\eta_{i+1}(\theta) \\
\eta_{i+2}(\theta) \\
\vdots \\
\eta_{i+m-1}(\theta)
\end{array}\right]
$$

Moreover, the entries of the Fisher information matrix satisfy

$$
g_{i j}(\theta)=\eta_{i+j}(\theta)-\eta_{i}(\theta) \eta_{j}(\theta) .
$$

Proof : All results, excepted (5), may be found or immediately derived from [1]. We only notice that some of the above properties follow easily by differentiating the identity

$$
\int \exp \left[\theta^{T} c(x)-\psi(\theta)\right] d x=1
$$

RR $\mathrm{n}^{\circ} 2598$ 
w.r.t. the components $\left(\theta_{1}, \cdots, \theta_{m}\right)$ of $\theta$. The particular recursion formula (5) is obtained via the following integration by parts:

$$
\begin{aligned}
\eta_{i}(\theta) & =\int_{-\infty}^{+\infty} x^{i} p(x, \theta) d x \\
& =\left[\frac{x^{i+1}}{i+1} p(x, \theta)\right]_{-\infty}^{+\infty}-\int_{-\infty}^{+\infty} \frac{x^{i+1}}{i+1}\left[\theta_{1}+2 \theta_{2} x+\cdots+m \theta_{m} x^{m-1}\right] p(x, \theta) d x \\
& =0-\frac{1}{i+1} E_{p(, \theta)}\left\{\theta_{1} x^{i+1}+2 \theta_{2} x^{i+2}+\cdots+m \theta_{m} x^{i+m}\right\}
\end{aligned}
$$

from which the formula follows easily, remembering that $\eta_{i}(\theta)=E_{p(\cdot, \theta)}\left\{x^{i}\right\}$.

Remark 2.4 The quantities

$$
\left(\eta_{1}, \cdots, \eta_{m}\right) \in \mathcal{E}=\eta(\Theta) \subset \mathbf{R}^{m}
$$

form a coordinate system for the given exponential family. The two coordinate systems $\theta$ (canonical parameters) and $\eta$ (expectation parameters) are related by diffeomorphism, and according to the above results the Jacobian matrix of the transformation $\eta=\eta(\theta)$ is the Fisher information matrix. We shall use the notation $p_{E}(\cdot, \eta(\theta))=p(\cdot, \theta)$ to express exponential densities of $S$ as functions of the expectation parameters.

The canonical parameters and the expectation parameters are biorthogonal w.r.t. the Fisher information metric : at $\sqrt{p(\cdot, \theta)}=\sqrt{p_{E}(\cdot, \eta)}$

$$
\left\langle\frac{\partial}{\partial \theta_{i}} \sqrt{p(\cdot, \theta)}, \frac{\partial}{\partial \eta_{j}} \sqrt{p_{E}(\cdot, \eta)}\right\rangle=\delta_{i j}, \quad i, j=1,2, \cdots, m .
$$

\section{The nonlinear filtering problem}

On the probability space $(\Omega, \mathcal{F}, P)$ with the filtration $\left\{\mathcal{F}_{t}, t \geq 0\right\}$ we consider the following state and observation equations, see [16], [3], [10]

$$
\begin{aligned}
d X_{t} & =f_{t}\left(X_{t}\right) d t+\sigma_{t}\left(X_{t}\right) d W_{t} \\
d Y_{t} & =h_{t}\left(X_{t}\right) d t+d V_{t} .
\end{aligned}
$$

These equations are Itô stochastic differential equations. In (7), the unobserved state process $\left\{X_{t}, t \geq 0\right\}$ and the observation process $\left\{Y_{t}, t \geq 0\right\}$ are taking values in $\mathbf{R}^{n}$ and $\mathbf{R}^{d}$ respectively, the noise processes $\left\{W_{t}, t \geq 0\right\}$ and $\left\{V_{t}, t \geq 0\right\}$ are two Brownian motions, taking values in $\mathbf{R}^{p}$ and $\mathbf{R}^{d}$ respectively, with covariance matrices $Q_{t}$ and $R_{t}$ respectively. 
We assume that $R_{t}$ is invertible for all $t \geq 0$, which implies that, without loss of generality, we can assume that $R_{t}=I$ for all $t \geq 0$. Finally, the initial state $X_{0}$ and the noise processes $\left\{W_{t}, t \geq 0\right\}$ and $\left\{V_{t}, t \geq 0\right\}$ are mutually independent.

We assume that the initial state $X_{0}$ has a density $p_{0}$ w.r.t. the Lebesgue measure $\lambda$ on $\mathbf{R}^{n}$, and has finite moments of any order, and we make the following assumptions on the coefficients $f_{t}, a_{t}:=\sigma_{t} Q_{t} \sigma_{t}^{T}$, and $h_{t}$ of the system ( 7 )

(A) Local Lipschitz continuity : for all $R>0$, there exists $K_{R}>0$ such that

$$
\left|f_{t}(x)-f_{t}\left(x^{\prime}\right)\right| \leq K_{R}\left|x-x^{\prime}\right| \quad \text { and } \quad|| a_{t}(x)-a_{t}\left(x^{\prime}\right) \| \leq K_{R}\left|x-x^{\prime}\right|,
$$

for all $t \geq 0$, and for all $x, x^{\prime} \in B_{R}$, the ball of radius $R$.

(B) Non-explosion : there exists $K>0$ such that

$$
x^{T} f_{t}(x) \leq K\left(1+|x|^{2}\right) \quad \text { and } \quad \text { trace } a_{t}(x) \leq K\left(1+|x|^{2}\right),
$$

for all $t \geq 0$, and for all $x \in \mathbf{R}^{m}$.

(C) Polynomial growth : there exist $K>0$ and $r \geq 0$ such that

$$
\left|h_{t}(x)\right| \leq K\left(1+|x|^{r}\right),
$$

for all $t \geq 0$, and for all $x \in \mathbf{R}^{m}$.

Under assumptions (A) and (B), there exists a unique solution $\left\{X_{t}, t \geq 0\right\}$ to the state equation, see [11], and $X_{t}$ has finite moments of any order. Under the additional assumption (C) the following finite energy condition holds

$$
E \int_{0}^{T}\left|h_{t}\left(X_{t}\right)\right|^{2} d t<\infty, \quad \text { for all } T \geq 0 .
$$

The nonlinear filtering problem consists in finding the conditional probability distribution $\pi_{t}$ of the state $X_{t}$ given the observations up to time $t$, i.e. $\pi_{t}(d x):=P\left[X_{t} \in d x \mid \mathcal{Y}_{t}\right]$, where $\mathcal{Y}_{t}:=\sigma\left(Y_{s}, 0 \leq s \leq t\right)$. Since the finite energy condition holds, it follows from [5] that $\left\{\pi_{t}, t \geq 0\right\}$ satisfies the Kushner-Stratonovich equation, i.e. for any smooth and compactly supported test function $\phi$ defined on $\mathbf{R}^{n}$

$$
\pi_{t}(\phi)=\pi_{0}(\phi)+\int_{0}^{t} \pi_{s}\left(\mathcal{L}_{s} \phi\right) d s+\sum_{k=1}^{d} \int_{0}^{t}\left[\pi_{s}\left(h_{s}^{k} \phi\right)-\pi_{s}\left(h_{s}^{k}\right) \pi_{s}(\phi)\right]\left[d Y_{s}^{k}-\pi_{s}\left(h_{s}^{k}\right) d s\right],
$$

where for all $t \geq 0$, the backward diffusion operator $\mathcal{L}_{t}$ is defined by

$$
\mathcal{L}_{t}=\sum_{i=1}^{n} f_{t}^{i} \frac{\partial}{\partial x_{i}}+\frac{1}{2} \sum_{i, j=1}^{n} a_{t}^{i j} \frac{\partial^{2}}{\partial x_{i} \partial x_{j}}
$$

RR $n^{\circ} 2598$ 
The MFS form of equation (8) is

$$
\begin{aligned}
\pi_{t}(\phi)=\pi_{0}(\phi) & +\int_{0}^{t} \pi_{s}\left(\mathcal{L}_{s} \phi\right) d s-\frac{1}{2} \int_{0}^{t}\left[\pi_{s}\left(\left|h_{s}\right|^{2} \phi\right)-\pi_{s}\left(\left|h_{s}\right|^{2}\right) \pi_{s}(\phi)\right] d s \\
& +\sum_{k=1}^{d} \int_{0}^{t}\left[\pi_{s}\left(h_{s}^{k} \phi\right)-\pi_{s}\left(h_{s}^{k}\right) \pi_{s}(\phi)\right] \circ d Y_{s}^{k} .
\end{aligned}
$$

From now on we proceed formally, and we assume that for all $t \geq 0$, the probability distribution $\pi_{t}$ has a density $p_{t}$ w.r.t. the Lebesgue measure on $\mathbf{R}^{n}$. Then $\left\{p_{t}, t \geq 0\right\}$ satisfies

$$
d p_{t}=\mathcal{L}_{t}^{*} p_{t} d t+\sum_{k=1}^{d} p_{t}\left[h_{t}^{k}-E_{p_{t}}\left\{h_{t}^{k}\right\}\right]\left[d Y_{t}^{k}-E_{p_{t}}\left\{h_{t}^{k}\right\} d t\right]
$$

in a suitable functional space, where $E_{p_{t}}\{\cdot\}$ denotes the expectation w.r.t. the probability density $p_{t}$, i.e. the conditional expectation given the observations up to time $t$, and where for all $t \geq 0$, the forward diffusion operator $\mathcal{L}_{t}^{*}$ is defined by

$$
\mathcal{L}_{t}^{*} \phi=-\sum_{i=1}^{n} \frac{\partial}{\partial x_{i}}\left[f_{t}^{i} \phi\right]+\frac{1}{2} \sum_{i, j=1}^{n} \frac{\partial^{2}}{\partial x_{i} \partial x_{j}}\left[a_{t}^{i j} \phi\right],
$$

for any test function $\phi$ defined on $\mathbf{R}^{n}$. The corresponding MFS form of equation (10) is :

$$
d p_{t}=\mathcal{L}_{t}^{*} p_{t} d t-\frac{1}{2} p_{t}\left[\left|h_{t}\right|^{2}-E_{p_{t}}\left\{\left|h_{t}\right|^{2}\right\}\right] d t+\sum_{k=1}^{d} p_{t}\left[h_{t}^{k}-E_{p_{t}}\left\{h_{t}^{k}\right\}\right] \circ d Y_{t}^{k} .
$$

In order to simplify notation, we introduce the following definitions, which will be used throughout this paper :

$$
\begin{aligned}
& \alpha_{t}(p):=\frac{\mathcal{L}_{t}^{*} p}{p}, \beta_{t}^{0}(p):=\frac{1}{2}\left[\left|h_{t}\right|^{2}-E_{p}\left\{\left|h_{t}\right|^{2}\right\}\right], \\
& \beta_{t}^{k}(p):=h_{t}^{k}-E_{p}\left\{h_{t}^{k}\right\},
\end{aligned}
$$

for $k=1, \cdots, d$. Simple calculations show that

$$
\begin{aligned}
& \alpha_{t}(p)=-\sum_{i=1}^{n}\left[f_{t}^{i} \frac{\partial}{\partial x_{i}}(\log p)+\frac{\partial f_{t}^{i}}{\partial x_{i}}\right] \\
& +\frac{1}{2} \sum_{i, j=1}^{n}\left[a_{t}^{i j} \frac{\partial^{2}}{\partial x_{i} \partial x_{j}}(\log p)+a_{t}^{i j} \frac{\partial}{\partial x_{i}}(\log p) \frac{\partial}{\partial x_{j}}(\log p)+2 \frac{\partial a_{t}^{i j}}{\partial x_{j}} \frac{\partial}{\partial x_{i}}(\log p)+\frac{\partial^{2} a_{t}^{i j}}{\partial x_{i} \partial x_{j}}\right] .
\end{aligned}
$$


The MFS form of the Kushner-Stratonovich equation reads now

$$
d p_{t}=\mathcal{L}_{t}^{*} p_{t} d t-p_{t} \beta_{t}^{0}\left(p_{t}\right) d t+\sum_{k=1}^{d} p_{t} \beta_{t}^{k}\left(p_{t}\right) \circ d Y_{t}^{k}
$$

We shall frequently work with square roots of densities, rather than densities themselves. Then, we compute by formal rules, using the MFS form :

$$
\begin{aligned}
d \sqrt{p_{t}}=\frac{1}{2 \sqrt{p_{t}}} \circ d p_{t} & =\frac{1}{2} \sqrt{p_{t}} \alpha_{t}\left(p_{t}\right) d t-\frac{1}{2} \sqrt{p_{t}} \beta_{t}^{0}\left(p_{t}\right) d t+\frac{1}{2} \sum_{k=1}^{d} \sqrt{p_{t}} \beta_{t}^{k}\left(p_{t}\right) \circ d Y_{t}^{k} \\
& =\mathcal{P}_{t}\left(\sqrt{p_{t}}\right) d t-\mathcal{Q}_{t}^{0}\left(\sqrt{p_{t}}\right) d t+\sum_{k=1}^{d} \mathcal{Q}_{t}^{k}\left(\sqrt{p_{t}}\right) \circ d Y_{t}^{k}
\end{aligned}
$$

where the nonlinear time dependent operators $\mathcal{P}_{t}$ and $\mathcal{Q}_{t}^{k}$ for $k=0,1, \cdots, d$ are defined by

$$
\mathcal{P}_{t}(r):=\frac{1}{2} r \alpha_{t}\left(r^{2}\right)=\frac{\mathcal{L}_{t}^{*} r^{2}}{2 r}, \quad \mathcal{Q}_{t}^{k}(r):=\frac{1}{2} r \beta_{t}^{k}\left(r^{2}\right)
$$

respectively. Closed form solutions of the Kushner-Stratonovich equation are rarely found - for a discussion see e.g. [15]. Instead many possible schemes for approximate nonlinear filters have been constructed, like the extended Kalman filter (EKF) or the assumed density filters (ADF). Now that we have briefly stated the nonlinear filtering problem, how does differential geometry enter the picture?

\section{The exponential projection filter}

In this section we present the rigorous definition of an exponential projection filter. We will show that if we choose $S^{1 / 2}$ as the set of square roots of probability densities of a finite dimensional exponential family, then under an additional assumption, see (18) below, the operators $\mathcal{P}_{t}$ and $\mathcal{Q}_{t}^{k}$ for $k=0,1, \cdots, d$, introduced in (14) map elements of $S^{1 / 2}$ into $L_{2}(\lambda)$. This is important because in general the operator $\mathcal{P}_{t}$ is unbounded, i.e. does not map $L_{2}(\lambda)$ into $L_{2}(\lambda)$, and the projection, according to formula (3), of the coefficients in the right hand side of the Kushner-Stratonovich equation is not possible. Let us consider the following exponential family of probability densities

$$
S:=\{p(\cdot, \theta), \theta \in \Theta\}, \quad p(x, \theta):=\exp \left[\theta^{T} c(x)-\psi(\theta)\right] .
$$

According to (11), we define for all $\theta \in \Theta$, and all $t \geq 0$

$$
\begin{aligned}
\alpha_{t, \theta}:=\alpha_{t}(p(\cdot, \theta))=\frac{\mathcal{L}_{t}^{*} p(\cdot, \theta)}{p(\cdot, \theta)}, \quad \beta_{t, \theta}^{0}:=\beta_{t}^{0}(p(\cdot, \theta))=\frac{1}{2}\left[\left|h_{t}\right|^{2}-E_{p(\cdot, \theta)}\left\{\left|h_{t}\right|^{2}\right\}\right] \\
\beta_{t, \theta}^{k}:=\beta_{t}^{k}(p(\cdot, \theta))=\left[h_{t}^{k}-E_{p(\cdot, \theta)}\left\{h_{t}^{k}\right\}\right],
\end{aligned}
$$

RR $\mathrm{n}^{\circ} 2598$ 
for $k=1, \cdots, d$. From the expression obtained in (12), it follows that

$$
\begin{aligned}
& \alpha_{t, \theta}=-\sum_{i=1}^{n}\left[f_{t}^{i} \frac{\partial}{\partial x_{i}}\left(\theta^{T} c\right)+\frac{\partial f_{t}^{i}}{\partial x_{i}}\right] \\
& +\frac{1}{2} \sum_{i, j=1}^{n}\left[a_{t}^{i j} \frac{\partial^{2}}{\partial x_{i} \partial x_{j}}\left(\theta^{T} c\right)+a_{t}^{i j} \frac{\partial}{\partial x_{i}}\left(\theta^{T} c\right) \frac{\partial}{\partial x_{j}}\left(\theta^{T} c\right)+2 \frac{\partial a_{t}^{i j}}{\partial x_{j}} \frac{\partial}{\partial x_{i}}\left(\theta^{T} c\right)+\frac{\partial^{2} a_{t}^{i j}}{\partial x_{i} \partial x_{j}}\right] .
\end{aligned}
$$

We assume that for all $\theta \in \Theta$, and all $t \geq 0$

$$
E_{p(\cdot, \theta)}\left\{\left|\alpha_{t, \theta}\right|^{2}\right\}<\infty \quad \text { and } \quad E_{p(\cdot, \theta)}\left\{\left|h_{t}\right|^{4}\right\}<\infty \text {, }
$$

which implies that $\mathcal{P}_{t}(\sqrt{p(\cdot, \theta)})$ and $Q_{t}^{k}(\sqrt{p(\cdot, \theta)})$ for $k=0,1, \cdots, d$ are vectors of $L_{2}(\lambda)$ for all $\theta \in \Theta$, and all $t \geq 0$.

Let us consider the equation (13) in MFS form for $\left\{\sqrt{p_{t}}, t \geq t_{0}\right\}$, starting at time $t_{0}$ from the initial condition $\sqrt{p_{t_{0}}}=\sqrt{p\left(\cdot, \theta_{0}\right)} \in S^{1 / 2}$ for some $\theta_{0} \in \Theta$, i.e.

$$
d \sqrt{p_{t}}=\mathcal{P}_{t}\left(\sqrt{p_{t}}\right) d t-\mathcal{Q}_{t}^{0}\left(\sqrt{p_{t}}\right) d t+\sum_{k=1}^{d} \mathcal{Q}_{t}^{k}\left(\sqrt{p_{t}}\right) \circ d Y_{t}^{k}, \quad t \geq t_{0}
$$

Under our assumptions, $\mathcal{P}_{t_{0}}\left(\sqrt{p_{t_{0}}}\right)$ and $Q_{t_{0}}^{k}\left(\sqrt{p_{t_{0}}}\right)$ for $k=0,1, \cdots, d$ are vectors of $L_{2}(\lambda)$, which we can project onto the finite dimensional tangent vector space $L \sqrt{p\left(;, \theta_{0}\right)} S^{1 / 2}$. For this purpose, we define for all $\theta \in \Theta$ the orthogonal projection

$$
\begin{aligned}
\Pi_{\theta}: L_{2}(\lambda) & \longrightarrow L_{\sqrt{p(\cdot \theta)}} S^{1 / 2} \\
v & \longmapsto \sum_{i=1}^{m}\left[\sum_{j=1}^{m} 4 g^{i j}(\theta)\left\langle v, \frac{1}{2 \sqrt{p(\cdot, \theta)}} \frac{\partial p(\cdot, \theta)}{\partial \theta_{j}}\right\rangle\right] \frac{1}{2 \sqrt{p(\cdot, \theta)}} \frac{\partial p(\cdot, \theta)}{\partial \theta_{i}} .
\end{aligned}
$$

The exponential projection filter for the exponential family $S$ is defined as the solution of the following stochastic differential equation on the manifold $S^{1 / 2}$ :

$$
\begin{aligned}
d \sqrt{p\left(\cdot, \theta_{t}\right)}= & \Pi_{\theta_{t}} \circ \mathcal{P}_{t}\left(\sqrt{p\left(\cdot, \theta_{t}\right)}\right) d t \\
& -\Pi_{\theta_{t}} \circ \mathcal{Q}_{t}^{0}\left(\sqrt{p\left(\cdot, \theta_{t}\right)}\right) d t+\sum_{k=1}^{d} \Pi_{\theta_{t}} \circ \mathcal{Q}_{t}^{k}\left(\sqrt{p\left(\cdot, \theta_{t}\right)}\right) \circ d Y_{t}^{k}
\end{aligned}
$$

Of course the operators

$$
\begin{aligned}
S^{1 / 2} & \longrightarrow L S^{1 / 2} \\
\sqrt{p(\cdot, \theta)} & \longmapsto \Pi_{\theta} \circ \mathcal{P}_{t}(\sqrt{p(\cdot, \theta)}) \in L_{\sqrt{p(\cdot, \theta)}} S^{1 / 2},
\end{aligned}
$$


and

$$
\begin{aligned}
S^{1 / 2} & \longrightarrow L S^{1 / 2} \\
\sqrt{p(\cdot, \theta)} & \longmapsto \Pi_{\theta} \circ \mathcal{Q}_{t}^{k}(\sqrt{p(\cdot, \theta)}) \in L_{\sqrt{p(\cdot, \theta)}} S^{1 / 2}
\end{aligned}
$$

for $k=0,1, \cdots, d$, are vector fields on the manifold $S^{1 / 2}$, for all $t \geq 0$. We can now state the following theorem :

Theorem 4.1 Assume that, in addition to (A), (B) and (C), the coefficients $f_{t}, a_{t}$ and $h_{t}$ of the system (7), and the coefficients $c$ of the exponential family (15) are such that:

$$
E_{p(\cdot, \theta)}\left\{\left|\alpha_{t, \theta}\right|^{2}\right\}<\infty \quad \text { and } \quad E_{p(\cdot, \theta)}\left\{\left|h_{t}\right|^{4}\right\}<\infty,
$$

holds for all $\theta \in \Theta$, and all $t \geq 0$, where the expression of $\alpha_{t, \theta}$ is given in (16).

Then, for $\theta \in \Theta$, and all $t>0, \Pi_{\theta} \circ \mathcal{P}_{t}$ and $\Pi_{\theta} \circ \mathcal{Q}_{t}^{k}$ for $k=0,1, \cdots, d$ are vector fields on the exponential manifold $S^{1 / 2}$.

The projection fllter density $p_{t}^{\pi}=p\left(\cdot, \theta_{t}\right)$ is described by equation (17), and the projection flter parameters satisfy the following stochastic differential equation:

$$
\begin{aligned}
g\left(\theta_{t}\right) \circ d \theta_{t}= & E_{p\left(\cdot, \theta_{t}\right)}\left\{\mathcal{L}_{t} c\right\} d t-E_{p\left(\cdot, \theta_{t}\right)}\left\{\frac{1}{2}\left|h_{t}\right|^{2}\left[c-\eta\left(\theta_{t}\right)\right]\right\} d t \\
& +\sum_{k=1}^{d} E_{p\left(\cdot, \theta_{t}\right)}\left\{h_{t}^{k}\left[c-\eta\left(\theta_{t}\right)\right]\right\} \circ d Y_{t}^{k}
\end{aligned}
$$

where the expectation parameters $\eta_{1}(\theta), \cdots, \eta_{m}(\theta)$, are defined in Lemma 2.3.

Under the assumptions on the coefficients, this equation has a unique solution, up to the a.s. positive time $\tau:=\inf \left\{t>0: \theta_{t} \notin \Theta\right\}$.

Remark 4.2 The question of whether the exit time $\tau$ is a.s. finite or infinite will be addressed elsewhere.

Remark 4.3 The weaker conditions

$$
E_{p(\cdot, \theta)}\left\{\left|\mathcal{L}_{t} c\right|\right\}<\infty \quad \text { and } \quad E_{p(\cdot, \theta)}\left\{\left|h_{t}\right|^{2}\right\}<\infty
$$

for all $\theta \in \Theta$, and all $t \geq 0$, are sufficient for proving existence and uniqueness of a solution to equation (19) up to the exit time $\tau$. The question of whether the interpretation as a projected equation still holds under these weaker conditions will require further investigation.

Proof : Let us compute

$$
\Pi_{\theta_{t}} \circ \mathcal{P}_{t}\left(\sqrt{p\left(\cdot, \theta_{t}\right)}\right)=\Pi_{\theta_{t}}\left[\frac{\mathcal{L}_{t}^{*} p\left(\cdot, \theta_{t}\right)}{2 \sqrt{p\left(\cdot, \theta_{t}\right)}}\right]=
$$




$$
\begin{aligned}
& =\sum_{i=1}^{m}\left[\sum_{j=1}^{m} 4 g^{i j}\left(\theta_{t}\right)\left\langle\frac{\mathcal{L}_{t}^{*} p\left(\cdot, \theta_{t}\right)}{2 \sqrt{p\left(\cdot, \theta_{t}\right)}}, \frac{1}{2 \sqrt{p\left(\cdot, \theta_{t}\right)}} \frac{\partial p\left(\cdot, \theta_{t}\right)}{\partial \theta_{j}}\right\rangle\right] \frac{1}{2 \sqrt{p\left(\cdot, \theta_{t}\right)}} \frac{\partial p\left(\cdot, \theta_{t}\right)}{\partial \theta_{i}} \\
& =\sum_{i=1}^{m}\left[\sum_{j=1}^{m} g^{i j}\left(\theta_{t}\right) \int \frac{\mathcal{L}_{t}^{*} p\left(x, \theta_{t}\right)}{p\left(x, \theta_{t}\right)} \frac{\partial p\left(x, \theta_{t}\right)}{\partial \theta_{j}} d \lambda(x)\right] \frac{1}{2 \sqrt{p\left(\cdot, \theta_{t}\right)}} \frac{\partial p\left(\cdot, \theta_{t}\right)}{\partial \theta_{i}} .
\end{aligned}
$$

Similarly

$$
\begin{aligned}
& \Pi_{\theta_{t}} \circ \mathcal{Q}_{t}^{0}\left(\sqrt{p\left(\cdot, \theta_{t}\right)}\right)=\Pi_{\theta_{t}}\left[\frac{1}{2} \sqrt{p\left(\cdot, \theta_{t}\right)} \beta_{t}^{0}\left(p\left(\cdot, \theta_{t}\right)\right)\right]= \\
= & \sum_{i=1}^{m}\left[\sum_{j=1}^{m} 4 g^{i j}\left(\theta_{t}\right)\left\langle\frac{1}{2} \sqrt{p\left(\cdot, \theta_{t}\right)} \beta_{t}^{0}\left(p\left(\cdot, \theta_{t}\right)\right), \frac{1}{2 \sqrt{p\left(\cdot, \theta_{t}\right)}} \frac{\partial p\left(\cdot, \theta_{t}\right)}{\partial \theta_{j}}\right\rangle\right] \frac{1}{2 \sqrt{p\left(\cdot, \theta_{t}\right)}} \frac{\partial p\left(\cdot, \theta_{t}\right)}{\partial \theta_{i}} \\
= & \sum_{i=1}^{m}\left[\sum_{j=1}^{m} g^{i j}\left(\theta_{t}\right) \int \frac{1}{2}\left[\left|h_{t}(x)\right|^{2}-E_{p\left(\cdot, \theta_{t}\right)}\left\{\left|h_{t}\right|^{2}\right\}\right] \frac{\partial p\left(x, \theta_{t}\right)}{\partial \theta_{j}} d \lambda(x)\right] \frac{1}{2 \sqrt{p\left(\cdot, \theta_{t}\right)}} \frac{\partial p\left(\cdot, \theta_{t}\right)}{\partial \theta_{i}} \\
= & \sum_{i=1}^{m}\left[\sum_{j=1}^{m} g^{i j}\left(\theta_{t}\right) \int \frac{1}{2}\left|h_{t}(x)\right|^{2} \frac{\partial p\left(x, \theta_{t}\right)}{\partial \theta_{j}} d \lambda(x)\right] \frac{1}{2 \sqrt{p\left(\cdot, \theta_{t}\right)}} \frac{\partial p\left(\cdot, \theta_{t}\right)}{\partial \theta_{i}}
\end{aligned}
$$

and

$$
\begin{aligned}
& \Pi_{\theta_{t}} \circ \mathcal{Q}_{t}^{k}\left(\sqrt{p\left(\cdot, \theta_{t}\right)}\right)=\Pi_{\theta_{t}}\left[\frac{1}{2} \sqrt{p\left(\cdot, \theta_{t}\right)} \beta_{t}^{k}\left(p\left(\cdot, \theta_{t}\right)\right)\right]= \\
= & \sum_{i=1}^{m}\left[\sum_{j=1}^{m} 4 g^{i j}\left(\theta_{t}\right)\left\langle\frac{1}{2} \sqrt{p\left(\cdot, \theta_{t}\right)} \beta_{t}^{k}\left(p\left(\cdot, \theta_{t}\right)\right), \frac{1}{2 \sqrt{p\left(\cdot, \theta_{t}\right)}} \frac{\partial p\left(\cdot, \theta_{t}\right)}{\partial \theta_{j}}\right\rangle\right] \frac{1}{2 \sqrt{p\left(\cdot, \theta_{t}\right)}} \frac{\partial p\left(\cdot, \theta_{t}\right)}{\partial \theta_{i}} \\
= & \sum_{i=1}^{m}\left[\sum_{j=1}^{m} g^{i j}\left(\theta_{t}\right) \int\left[h_{t}^{k}(x)-E_{p\left(\cdot, \theta_{t}\right)}\left\{h_{t}^{k}\right\}\right] \frac{\partial p\left(x, \theta_{t}\right)}{\partial \theta_{j}} d \lambda(x)\right] \frac{1}{2 \sqrt{p\left(\cdot, \theta_{t}\right)}} \frac{\partial p\left(\cdot, \theta_{t}\right)}{\partial \theta_{i}} \\
= & \sum_{i=1}^{m}\left[\sum_{j=1}^{m} g^{i j}\left(\theta_{t}\right) \int h_{t}^{k}(x) \frac{\partial p\left(x, \theta_{t}\right)}{\partial \theta_{j}} d \lambda(x)\right] \frac{1}{2 \sqrt{p\left(\cdot, \theta_{t}\right)}} \frac{\partial p\left(\cdot, \theta_{t}\right)}{\partial \theta_{i}}
\end{aligned}
$$

for $k=1, \cdots, d$. We have used the fact that the constant terms $E_{p\left(\cdot, \theta_{t}\right)}\left\{\left|h_{t}\right|^{2}\right\}$ and $E_{p\left(\cdot, \theta_{t}\right)}\left\{h_{t}^{k}\right\}$ give no contribution to the projection, since

$$
\int \frac{\partial p\left(x, \theta_{t}\right)}{\partial \theta_{i}} d \lambda(x)=0 .
$$


We conclude by rewriting equation (17) in the more detailed form

$$
\begin{aligned}
d & \sqrt{p\left(\cdot, \theta_{t}\right)}=\sum_{i=1}^{m}\left[\sum_{j=1}^{m} g^{i j}\left(\theta_{t}\right) \int \frac{\mathcal{L}_{t}^{*} p\left(x, \theta_{t}\right)}{p\left(x, \theta_{t}\right)} \frac{\partial p\left(x, \theta_{t}\right)}{\partial \theta_{j}} d \lambda(x)\right] \frac{1}{2 \sqrt{p\left(\cdot, \theta_{t}\right)}} \frac{\partial p\left(\cdot, \theta_{t}\right)}{\partial \theta_{i}} d t \\
& -\sum_{i=1}^{m}\left[\sum_{j=1}^{m} g^{i j}\left(\theta_{t}\right) \int \frac{1}{2}\left|h_{t}(x)\right|^{2} \frac{\partial p\left(x, \theta_{t}\right)}{\partial \theta_{j}} d \lambda(x)\right] \frac{1}{2 \sqrt{p\left(\cdot, \theta_{t}\right)}} \frac{\partial p\left(\cdot, \theta_{t}\right)}{\partial \theta_{i}} d t \\
& +\sum_{i=1}^{m} \sum_{k=1}^{d}\left[\sum_{j=1}^{m} g^{i j}\left(\theta_{t}\right) \int h_{t}^{k}(x) \frac{\partial p\left(x, \theta_{t}\right)}{\partial \theta_{j}} d \lambda(x)\right] \frac{1}{2 \sqrt{p\left(\cdot, \theta_{t}\right)}} \frac{\partial p\left(\cdot, \theta_{t}\right)}{\partial \theta_{i}} \circ d Y_{t}^{k}
\end{aligned}
$$

By expanding $\sqrt{p\left(\cdot, \theta_{t}\right)}$ according to

$$
d \sqrt{p\left(\cdot, \theta_{t}\right)}=\sum_{i=1}^{m} \frac{1}{2 \sqrt{p\left(\cdot, \theta_{t}\right)}} \frac{\partial p\left(\cdot, \theta_{t}\right)}{\partial \theta_{i}} \circ d \theta_{t}^{i},
$$

and comparing with (20) we obtain the following equation for the parameters $\theta_{t}$ describing our projected density in $S$ :

$$
\begin{aligned}
d \theta_{t}^{i}= & {\left[\sum_{j=1}^{m} g^{i j}\left(\theta_{t}\right) \int \frac{\mathcal{L}_{t}^{*} p\left(x, \theta_{t}\right)}{p\left(x, \theta_{t}\right)} \frac{\partial p\left(x, \theta_{t}\right)}{\partial \theta_{j}} d \lambda(x)\right] d t } \\
& -\left[\sum_{j=1}^{m} g^{i j}\left(\theta_{t}\right) \int \frac{1}{2}\left|h_{t}(x)\right|^{2} \frac{\partial p\left(x, \theta_{t}\right)}{\partial \theta_{j}} d \lambda(x)\right] d t \\
& +\sum_{k=1}^{d}\left[\sum_{j=1}^{m} g^{i j}\left(\theta_{t}\right) \int h_{t}^{k}(x) \frac{\partial p\left(x, \theta_{t}\right)}{\partial \theta_{j}} d \lambda(x)\right] \circ d Y_{t}^{k},
\end{aligned}
$$

which is our finite dimensional filter. This holds formally for any manifold $S^{1 / 2}$, even if $S$ is not an exponential family. For the special case of the exponential family introduced above in (15), we obtain

$$
\begin{aligned}
d \theta_{t}^{i}= & {\left[\sum_{j=1}^{m} g^{i j}\left(\theta_{t}\right) \int \mathcal{L}_{t} c_{j}(x) p\left(x, \theta_{t}\right) d \lambda(x)\right] d t } \\
& -\left[\sum_{j=1}^{m} g^{i j}\left(\theta_{t}\right) \int \frac{1}{2}\left|h_{t}(x)\right|^{2}\left[c_{j}(x)-\eta_{j}\left(\theta_{t}\right)\right] p\left(x, \theta_{t}\right) d \lambda(x)\right] d t \\
& +\sum_{k=1}^{d}\left[\sum_{j=1}^{m} g^{i j}\left(\theta_{t}\right) \int h_{t}^{k}(x)\left[c_{j}(x)-\eta_{j}\left(\theta_{t}\right)\right] p\left(x, \theta_{t}\right) d \lambda(x)\right] \circ d Y_{t}^{k}
\end{aligned}
$$


We have used the following duality relation

$$
\begin{aligned}
\int \frac{\mathcal{L}_{t}^{*} p\left(x, \theta_{t}\right)}{p\left(x, \theta_{t}\right)} \frac{\partial p\left(x, \theta_{t}\right)}{\partial \theta_{j}} d \lambda(x) & =\int \mathcal{L}_{t}^{*} p\left(x, \theta_{t}\right)\left[c_{j}(x)-\eta_{j}\left(\theta_{t}\right)\right] d \lambda(x) \\
& =\int \mathcal{L}_{t} c_{j}(x) p\left(x, \theta_{t}\right) d \lambda(x) .
\end{aligned}
$$

Another way of writing equation (21) is

$$
\begin{aligned}
d \theta_{t}^{i}= & {\left[\sum_{j=1}^{m} g^{i j}\left(\theta_{t}\right) E_{p\left(\cdot, \theta_{t}\right)}\left\{\mathcal{L}_{t} c_{j}\right\}\right] d t-\left[\sum_{j=1}^{m} g^{i j}\left(\theta_{t}\right) E_{p\left(\cdot, \theta_{t}\right)}\left\{\frac{1}{2}\left|h_{t}\right|^{2}\left[c_{j}-\eta_{j}\left(\theta_{t}\right)\right]\right\}\right] d t } \\
& +\sum_{k=1}^{d}\left[\sum_{j=1}^{m} g^{i j}\left(\theta_{t}\right) E_{p\left(\cdot, \theta_{t}\right)}\left\{h_{t}^{k}\left[c_{j}-\eta_{j}\left(\theta_{t}\right)\right]\right\}\right] \circ d Y_{t}^{k} .
\end{aligned}
$$

In vector form, the above equation reads

$$
\begin{aligned}
d \theta_{t}= & {\left[g\left(\theta_{t}\right)\right]^{-1} E_{p\left(\cdot, \theta_{t}\right)}\left\{\mathcal{L}_{t} c\right\} d t-\left[g\left(\theta_{t}\right)\right]^{-1} E_{p\left(\cdot, \theta_{t}\right)}\left\{\frac{1}{2}\left|h_{t}\right|^{2}\left[c-\eta\left(\theta_{t}\right)\right]\right\} d t } \\
& +\left[g\left(\theta_{t}\right)\right]^{-1} \sum_{k=1}^{d} E_{p\left(\cdot, \theta_{t}\right)}\left\{h_{t}^{k}\left[c-\eta\left(\theta_{t}\right)\right]\right\} \circ d Y_{t}^{k}
\end{aligned}
$$

or equivalently

$$
\begin{aligned}
g\left(\theta_{t}\right) \circ d \theta_{t}= & E_{p\left(\cdot, \theta_{t}\right)}\left\{\mathcal{L}_{t} c\right\} d t-E_{p\left(\cdot, \theta_{t}\right)}\left\{\frac{1}{2}\left|h_{t}\right|^{2}\left[c-\eta\left(\theta_{t}\right)\right]\right\} d t \\
& +\sum_{k=1}^{d} E_{p\left(\cdot, \theta_{t}\right)}\left\{h_{t}^{k}\left[c-\eta\left(\theta_{t}\right)\right]\right\} \circ d Y_{t}^{k} .
\end{aligned}
$$

Under the assumptions, the mappings $\theta \mapsto E_{p(, \theta)}\left\{\mathcal{L}_{t} c\right\}$, and $\theta \mapsto E_{p(\cdot, \theta)}\left\{\frac{1}{2}\left|h_{t}\right|^{2}\right\}$ are locally Lipschitz continuous. Therefore, there exists a unique solution to equation (19) up to the a.s. positive exit time $\tau$, see [11].

Remark 4.4 The initial condition $\theta_{0}$ for equation (19) is defined as follows : If $p_{0} \in S$, then $p_{0}=p\left(\cdot, \theta_{0}\right)$ for some unique $\theta_{0} \in \Theta$, which is used as the initial condition. Otherwise, we project $p_{0}$ on $S$, by minimizing the Kullback-Leibler information

$$
K\left(p_{0}, p(\cdot, \theta)\right):=\int \log \frac{p_{0}(x)}{p(x, \theta)} p_{0}(x) d \lambda(x),
$$

w.r.t. $\theta \in \Theta$. After straightforward calculations, and making use of Lemma 2.3, this reduces to maximizing

$$
\left[\theta^{T} \int c(x) p_{0}(x) d \lambda(x)-\psi(\theta)\right]
$$


Assuming the maximum is achieved in $\theta_{0} \in \Theta$, necessary conditions yield

$$
\eta_{i}\left(\theta_{0}\right)=\int c_{i}(x) p_{0}(x) d \lambda(x), \quad i=1, \cdots, m .
$$

\section{The relationship with the assumed density principle}

In the previous section we gave the formula for the exponential projection filter expressed in terms of the canonical parameters $\theta$. We present below a characterization of the exponential projection filter based on the expectation parameters $\eta$.

We begin by stating the assumed density filter idea. Assume we are given an exponential family $S$ as in Lemma 2.3, i.e.

$$
S=\{p(\cdot, \theta), \theta \in \Theta\}=\left\{p_{E}(\cdot, \eta), \eta \in \mathcal{E}\right\}, \quad p(x, \theta):=\exp \left[\theta^{T} c(x)-\psi(\theta)\right],
$$

where $\Theta \subset \mathbf{R}^{m}$ is open. The $m$-dimensional vector $\eta=E_{p_{E}(\cdot, \eta)}\{c\}$ is the vector of $e x$ pectation parameters of the density $p_{E}(\cdot, \eta) \in S$. More generally, for any density $p$ the $m$-dimensional vector $E_{p}\{c\}$ is called the vector of $c$-moments of the density $p$.

We need first to derive, under additional assumptions, the equation for the conditional $c$-moments, i.e. the $c$-moments of the conditional probability distribution. Indeed, if in addition to (A), (B) and (C), the coefficients $c$ of the exponential family (15) and their derivatives up to order 2 , and the coefficients $f_{t}$ and $a_{t}$ of the system (7), have at most polynomial growth when $|x|$ goes to infinity, then the conditions given in [5] are fulfilled for the $c$-moments to satisfy (9), i.e.

$$
\begin{aligned}
d \pi_{t}(c)= & \pi_{t}\left(\mathcal{L}_{t} c\right) d t-\frac{1}{2}\left[\pi_{t}\left(\left|h_{t}\right|^{2} c\right)-\pi_{t}\left(\left|h_{t}\right|^{2}\right) \pi_{t}(c)\right] d t \\
& +\sum_{k=1}^{d}\left[\pi_{t}\left(h_{t}^{k} c\right)-\pi_{t}\left(h_{t}^{k}\right) \pi_{t}(c)\right] \circ d Y_{t}^{k} .
\end{aligned}
$$

Since in general the conditional probability distribution $\pi_{t}$ cannot be recovered from its $c^{-}$ moments only, the stochastic differential equations (23) do not form a closed set of equations. The MFS-based exponential assumed density filter (MFSADF) is obtained by replacing in $(23)$ :

- the conditional probability distribution $\pi_{t}$ with the approximate a.c. probability distribution with density $p_{E}\left(\cdot, \eta_{t}\right)$ w.r.t. the Lebesgue measure on $\mathbf{R}^{n}$,

- the $c$-moments $\pi_{t}(c)$ of the conditional probability distribution, with the expectation parameter $\eta_{t}$ of the approximate density $p_{E}\left(\cdot, \eta_{t}\right)$.

This results in the following finite dimensional filter

$$
\begin{aligned}
d \eta_{t}= & E_{p_{E}\left(\cdot, \eta_{t}\right)}\left\{\mathcal{L}_{t} c\right\} d t-E_{p_{E}\left(\cdot, \eta_{t}\right)}\left\{\frac{1}{2}\left|h_{t}\right|^{2}\left[c-\eta_{t}\right]\right\} d t \\
& +\sum_{k=1}^{d} E_{p_{E}\left(\cdot, \eta_{t}\right)}\left\{h_{t}^{k}\left[c-\eta_{t}\right]\right\} \circ d Y_{t}^{k}
\end{aligned}
$$


The substitutions described above express the assumed density filter idea : we close the set of equations for the $c$-moments of the conditional probability distribution by assuming that this probability distribution is absolutely continuous, and has a density in the exponential family $S$ which is characterized by such $c$-moments. This idea involves a logically inconsistent procedure : in general the above assumption does not hold. Even if the definition of the MFSbased assumed density filter seems in general logically inconsistent, the following theorem gives a geometric characterization of this filter, which is then well defined despite the above remarks.

Theorem 5.1 Under the assumptions of Theorem 4.1, for any exponential family $S$, the projection filter coincides with the MFS-based assumed density filter.

Proof : We start from equation (19) for the projection filter canonical parameters, i.e.

$$
\begin{aligned}
g\left(\theta_{t}\right) \circ d \theta_{t}= & E_{p\left(\cdot, \theta_{t}\right)}\left\{\mathcal{L}_{t} c\right\} d t-E_{p\left(\cdot, \theta_{t}\right)}\left\{\frac{1}{2}\left|h_{t}\right|^{2}\left[c-\eta\left(\theta_{t}\right)\right]\right\} d t \\
& +\sum_{k=1}^{d} E_{p\left(\cdot, \theta_{t}\right)}\left\{h_{t}^{k}\left[c-\eta\left(\theta_{t}\right)\right]\right\} \circ d Y_{t}^{k} .
\end{aligned}
$$

According to Remark 2.4, the expectation parameters can be expressed in terms of the canonical parameters as

$$
\eta_{i}=\eta_{i}(\theta)=E_{p(\cdot, \theta)}\left\{c_{i}\right\}=E_{p_{E}(\cdot, \eta)}\left\{c_{i}\right\}
$$

with derivatives

$$
\frac{\partial}{\partial \theta_{j}} \eta_{j}(\theta)=g_{i j}(\theta) .
$$

The chain rule for Stratonovich integrals immediately gives

$$
\begin{aligned}
d \eta_{t}= & g\left(\theta_{t}\right) \circ d \theta_{t}=E_{p_{E}\left(\cdot, \eta_{t}\right)}\left\{\mathcal{L}_{t} c\right\} d t-E_{p_{E}\left(\cdot, \eta_{t}\right)}\left\{\frac{1}{2}\left|h_{t}\right|^{2}\left[c-\eta_{t}\right]\right\} d t \\
& +\sum_{k=1}^{d} E_{p_{E}\left(\cdot, \eta_{t}\right)}\left\{h_{t}^{k}\left[c-\eta_{t}\right]\right\} \circ d Y_{t}^{k}
\end{aligned}
$$

which is exactly equation (24) obtained using the assumed density filter idea.

Remark 5.2 It is possible to give an alternative more geometrical proof of this equivalence result, based on biorthogonality relations between canonical and expectation parameters in an exponential family, see [8].

Finally, it is worth noticing that the MFS-based assumed density filter is not just a MFS version of the Itô-based assumed density filter : in general, the following procedure

(i) write the Itô equations for the $c$-moments of the conditional probability distribution $\pi_{t}$, 
(ii) replace the conditional probability distribution $\pi_{t}$ with the approximate a.c. probability distribution with density $p_{E}\left(\cdot, \eta_{t}\right)$, and the $c$-moments $\pi_{t}(c)$ of the conditional probability distribution $\pi_{t}$, with the expectation parameters $\eta_{t}$ of the approximate density $p_{E}\left(\cdot, \eta_{t}\right)$,

(iii) transform the resulting Itô stochastic differential equation for the expectation parameter $\eta_{t}$ into MFS stochastic differential equation,

will not result in equation (24), see [8] for an explicit example. Then, for a general exponential family $S$ the equivalence with the projection filter holds only for the MFS-based ADF. However, it can be shown that the MFS-based and the Itô-based ADF coincide for special choices of exponential family, which are introduced in the next section.

\section{The projection residual and the choice of a conve- nient exponential family}

In this section, we are interested in defining quantities which will provide a local measure of the quality of the projection filter approximation. Compare equation (13) for the (square root of the) true density $p_{t}$, i.e.

$$
d \sqrt{p_{t}}=\mathcal{P}_{t}\left(\sqrt{p_{t}}\right) d t-\mathcal{Q}_{t}^{0}\left(\sqrt{p_{t}}\right) d t+\sum_{k=1}^{d} \mathcal{Q}_{t}^{k}\left(\sqrt{p_{t}}\right) \circ d Y_{t}^{k}
$$

and equation (17) for the (square root of the) projection filter density $p_{t}^{\pi}=p\left(\cdot, \theta_{t}\right)$, i.e.

$$
d \sqrt{p_{t}^{\pi}}=\Pi_{\theta_{t}} \circ \mathcal{P}_{t}\left(\sqrt{p_{t}^{\pi}}\right) d t-\Pi_{\theta_{t}} \circ \mathcal{Q}_{t}^{0}\left(\sqrt{p_{t}^{\pi}}\right) d t+\sum_{k=1}^{d} \Pi_{\theta_{t}} \circ \mathcal{Q}_{t}^{k}\left(\sqrt{p_{t}^{\pi}}\right) \circ d Y_{t}^{k} .
$$

Two steps are involved in using the projection filter density $p_{t}^{\pi}$ as an approximation of the true density $p_{t}$ : We make a first approximation by evaluating the right-hand side of equation (25) at the current projection filter density $p_{t}^{\pi}$ and not at the true density $p_{t}$. Even with this approximation, the resulting coefficients $\mathcal{P}_{t}\left(\sqrt{p_{t}^{\pi}}\right)$ and $\mathcal{Q}_{t}^{k}\left(\sqrt{p_{t}^{\pi}}\right)$ for $k=0,1, \cdots, d$ would make the solution leave the manifold $S^{1 / 2}$, and we make a second approximation by projecting these coefficients on the linear space $L{\sqrt{p_{t}^{\pi}}} S^{1 / 2}$ via the projection mapping $\Pi_{\theta_{t}}$. In order to express the error occurring in the second approximation step at time $t$, we define the prediction residual operator $\mathcal{R}_{t}^{\bullet}$ and the correction residual operators $\mathcal{R}_{t}^{k}$ for $k=0,1, \cdots, d$ as follows :

$$
\begin{aligned}
\mathcal{R}_{t}^{\bullet} & :=\mathcal{P}_{t}-\Pi_{\theta_{t}} \circ \mathcal{P}_{t} \\
\mathcal{R}_{t}^{k} & :=\mathcal{Q}_{t}^{k}-\Pi_{\theta_{t}} \circ \mathcal{Q}_{t}^{k} .
\end{aligned}
$$

These operators, when applied to the square root of density $\sqrt{p_{t}^{\pi}}=\sqrt{p\left(\cdot, \theta_{t}\right)} \in S^{1 / 2}$ yield vectors of $L_{2}(\lambda)$. We call such vectors projection residuals: they give a local measure of the 
quality of the approximation involved in the projection filter. We can compute the norm of such vectors according to the norm $\|\cdot\|$ in $L_{2}(\lambda)$, and we define the prediction residual norm $r_{t}^{\bullet}$ and correction residual norms $r_{t}^{k}$ for $k=0,1, \cdots, d$ as follows :

$$
\begin{aligned}
r_{t}^{\bullet} & :=\left\|\mathcal{R}_{t}^{\bullet}\left(\sqrt{p_{t}^{\pi}}\right)\right\| \\
r_{t}^{k} & :=\left\|\mathcal{R}_{t}^{k}\left(\sqrt{p_{t}^{\pi}}\right)\right\| .
\end{aligned}
$$

However, we are still missing a single measure of the local error resulting from the projection. We define below a single residual operator, only in the case where $\mathcal{R}_{t}^{k}=0$ for all $t \geq 0$, and all $k=1, \cdots, d$. In this case, we define the total residual operator $\mathcal{R}_{t}^{*}$ as :

$$
\mathcal{R}_{t}^{*}:=\mathcal{R}_{t}^{\bullet}-\mathcal{R}_{t}^{0},
$$

and the corresponding total residual norm $r_{t}^{*}$ as :

$$
r_{t}^{*}:=\left\|\mathcal{R}_{t}^{*}\left(\sqrt{p_{t}^{\pi}}\right)\right\| .
$$

Notice that if in addition $\mathcal{R}_{t}^{0}=0$, then $r_{t}^{*}$ reduces to $r_{t}^{\bullet}$. In the next section we will introduce manifolds $S_{\bullet}^{1 / 2}$ and $S_{*}^{1 / 2}$ for which such a definition is applicable. Now we try to give some intuition for the above definition. Suppose we replace in equations (25) and (26) the observation $\left\{Y_{t}, t \geq 0\right\}$ with some smooth process $\left\{u_{t}, t \geq 0\right\}$, e.g. a regularized approximation, i.e. we consider the equations

$$
\frac{d}{d t} \sqrt{p_{t}}=\mathcal{P}_{t}\left(\sqrt{p_{t}}\right)-\mathcal{Q}_{t}^{0}\left(\sqrt{p_{t}}\right)+\sum_{k=1}^{d} \mathcal{Q}_{t}^{k}\left(\sqrt{p_{t}}\right) \dot{u}_{t}^{k}
$$

and

$$
\frac{d}{d t} \sqrt{p_{t}^{\pi}}=\Pi_{\theta_{t}} \circ \mathcal{P}_{t}\left(\sqrt{p_{t}^{\pi}}\right)-\Pi_{\theta_{t}} \circ \mathcal{Q}_{t}^{0}\left(\sqrt{p_{t}^{\pi}}\right)+\sum_{k=1}^{d} \Pi_{\theta_{t}} \circ \mathcal{Q}_{t}^{k}\left(\sqrt{p_{t}^{\pi}}\right) \dot{u}_{t}^{k}
$$

In this case, we can define a single residual operator expressing the difference between the rate of change in the smooth Kushner-Stratonovich equation (27) and the rate of change in the smooth projection filter equation (28), i.e.

$$
\mathcal{R}_{t}^{u}:=\mathcal{R}_{t}^{\bullet}-\mathcal{R}_{t}^{0}+\sum_{k=1}^{d} \mathcal{R}_{t}^{k} \dot{u}_{t}^{k} .
$$

Of course, if we return to the original situation, e.g. letting the regularized approximation $\left\{u_{t}, t \geq 0\right\}$ converge to the observation $\left\{Y_{t}, t \geq 0\right\}$, there is no limit to the smooth residual operator $\mathcal{R}_{t}^{u}$, unless $\mathcal{R}_{t}^{k}=0$ for all $t \geq 0$, and all $k=1, \cdots, d$. In this case only, we define the total residual operator $\mathcal{R}_{t}^{*}$ as above.

From now on, and throughout the paper, we assume for simplicity that $h_{t}(x)=h(x)$ does not depend explicitly on time. This is necessary in order to define the simplifying time invariant exponential families $S_{*}$ and $S_{*}$ below. 


\subsection{The exponential families $S_{*}$ and $S_{*}$}

Now we can state the following

Theorem 6.1 Let $s:=\operatorname{rank}\left\{h^{1}, \cdots, h^{d}, \frac{1}{2}|h|^{2}\right\} \leq d+1$. There exist s linearly independent functions $\left\{c_{1}, \cdots, c_{s}\right\}$ defined on $\mathbf{R}^{n}$, such that for all $x \in \mathbf{R}^{n}$

$$
\frac{1}{2}|h(x)|^{2}=\sum_{i=1}^{s} \lambda_{i}^{0} c_{i}(x), \quad h^{k}(x)=\sum_{i=1}^{s} \lambda_{i}^{k} c_{i}(x),
$$

for $k=1, \cdots, d$. Remaining functions $\left\{c_{s+1}, \cdots, c_{m}\right\}$ are chosen such that

$$
S_{\bullet}:=\{p(\cdot, \theta), \theta \in \Theta\}, \quad p(x, \theta):=\exp \left[\theta^{T} c(x)-\psi(\theta)\right],
$$

where $\Theta \subseteq \mathbf{R}^{m}$ is open, is an exponential family of probability densities.

Assume that, in addition to (A), (B) and (C), the coefficients $f_{t}$ and $a_{t}$ of the system (7), and the coefficients $c$ of the exponential family $S_{\text {. are such that: }}$

$$
E_{p(\cdot, \theta)}\left\{\left|\alpha_{t, \theta}\right|^{2}\right\}<\infty
$$

holds for all $\theta \in \Theta$, and all $t \geq 0$, where the expression of $\alpha_{t, \theta}$ is given in (16).

Then, for the projection filter associated with the exponential family $S_{\bullet}$, the correction residual norms $r_{t}^{k}$ are identically zero for all $t \geq 0$, and all $k=0,1, \cdots, d$, and the stochastic differential equation for the parameters reduces to:

$$
d \theta_{t}=\left[g\left(\theta_{t}\right)\right]^{-1} E_{p\left(\cdot, \theta_{t}\right)}\left\{\mathcal{L}_{t} c\right\} d t-\lambda_{\bullet}^{0} d t+\sum_{k=1}^{d} \lambda_{\bullet}^{k} d Y_{t}^{k}
$$

where for all $k=0,1, \cdots, d$ the $m$-dimensional vector $\lambda_{\bullet}^{k}$ is defined by

$$
\lambda_{\bullet}^{k}=\left[\begin{array}{c}
\lambda_{1}^{k} \\
\vdots \\
\lambda_{s}^{k} \\
0 \\
\vdots \\
0
\end{array}\right]
$$

Under the assumptions on the coefficients, this equation has a unique solution, up to the a.s. positive exit time $\tau:=\inf \left\{t>0: \theta_{t} \notin \Theta\right\}$.

Proof : All the assumptions of Theorem 4.1 are satisfied, and therefore the solution of the stochastic differential equation for the projection filter with manifold $S_{\bullet}^{1 / 2}$ exists and is unique up to the a.s. positive exit time $\tau$.

$\operatorname{RR} \mathrm{n}^{\circ} 2598$ 
Next, we prove that the correction residual norms vanish. Indeed, it follows from (29) that

$$
\begin{aligned}
\mathcal{Q}_{t}^{0}\left(\sqrt{p\left(\cdot, \theta_{t}\right)}\right) & =\frac{1}{4}\left[|h|^{2}-E_{p\left(\cdot, \theta_{t}\right)}\left\{|h|^{2}\right\}\right] \sqrt{p\left(\cdot, \theta_{t}\right)} \\
& =\frac{1}{2} \sum_{i=1}^{s} \lambda_{i}^{0}\left[c_{i}-E_{p\left(\cdot, \theta_{t}\right)}\left\{c_{i}\right\}\right] \sqrt{p\left(\cdot, \theta_{t}\right)}
\end{aligned}
$$

and similarly

$$
\begin{aligned}
\mathcal{Q}_{t}^{k}\left(\sqrt{p\left(\cdot, \theta_{t}\right)}\right) & =\frac{1}{2}\left[h^{k}-E_{p\left(\cdot, \theta_{t}\right)}\left\{h^{k}\right\}\right] \sqrt{p\left(\cdot, \theta_{t}\right)} \\
& =\frac{1}{2} \sum_{i=1}^{s} \lambda_{i}^{k}\left[c_{i}-E_{p\left(\cdot, \theta_{t}\right)}\left\{c_{i}\right\}\right] \sqrt{p\left(\cdot, \theta_{t}\right)}
\end{aligned}
$$

for $k=1, \cdots, d$. We remark that

$$
\frac{1}{2}\left[c_{i}-E_{p\left(\cdot, \theta_{t}\right)}\left\{c_{i}\right\}\right] \sqrt{p\left(\cdot, \theta_{t}\right)}=\frac{1}{2}\left[c_{i}-\eta_{i}\left(\theta_{t}\right)\right] \sqrt{p\left(\cdot, \theta_{t}\right)}=\frac{1}{2 \sqrt{p\left(\cdot, \theta_{t}\right)}} \frac{\partial p\left(\cdot, \theta_{t}\right)}{\partial \theta_{i}},
$$

hence $\mathcal{Q}_{t}^{k}\left(\sqrt{p\left(\cdot, \theta_{t}\right)}\right) \in L_{\sqrt{p\left(\cdot, \theta_{t}\right)}} S^{1 / 2}$ for $k=0,1, \cdots, d$. Therefore, the projection does not modify these vectors since they already lie in the tangent space of $S^{1 / 2}$.

Finally, the equation for the parameters is obtained via straightforward calculations. Indeed, it follows from (29) that

$$
E_{p\left(, \theta_{t}\right)}\left\{\frac{1}{2}|h|^{2}\left[c_{j}-\eta_{j}\left(\theta_{t}\right)\right]\right\}=\sum_{l=1}^{s} \lambda_{l}^{0} E_{p\left(\cdot, \theta_{i}\right)}\left\{c_{l}\left[c_{j}-\eta_{j}\left(\theta_{t}\right)\right]\right\}=\sum_{l=1}^{s} g_{j l}\left(\theta_{t}\right) \lambda_{l}^{0},
$$

hence

$$
\sum_{j=1}^{m} g^{i j}\left(\theta_{t}\right) E_{p\left(\cdot, \theta_{t}\right)}\left\{\frac{1}{2}|h|^{2}\left[c_{j}-\eta_{j}\left(\theta_{t}\right)\right]\right\}=\sum_{j=1}^{m} g^{i j}\left(\theta_{t}\right) \sum_{l=1}^{s} g_{j l}\left(\theta_{t}\right) \lambda_{l}^{0}=\sum_{l=1}^{s} \delta_{i l} \lambda_{l}^{0},
$$

and similarly

$$
\sum_{j=1}^{m} g^{i j}\left(\theta_{t}\right) E_{p\left(\cdot, \theta_{t}\right)}\left\{h^{k}\left[c_{j}-\eta_{j}\left(\theta_{t}\right)\right]\right\}=\sum_{l=1}^{s} \delta_{i l} \lambda_{l}^{k},
$$

for all $k=1, \cdots, d$. Substituting these expressions into the right-hand side of equation (22) yields

$$
d \theta_{t}^{i}=\left[\sum_{j=1}^{m} g^{i j}\left(\theta_{t}\right) E_{p\left(\cdot, \theta_{t}\right)}\left\{\mathcal{L}_{t} c_{j}\right\}\right] d t-\left[\sum_{l=1}^{s} \delta_{i l} \lambda_{l}^{0}\right] d t+\sum_{k=1}^{d}\left[\sum_{l=1}^{s} \delta_{i l} \lambda_{l}^{k}\right] d Y_{t}^{k} .
$$

In vector form, the above equation reads

$$
d \theta_{t}=\left[g\left(\theta_{t}\right)\right]^{-1} E_{p\left(\cdot, \theta_{t}\right)}\left\{\mathcal{L}_{t} c\right\} d t-\lambda_{\bullet}^{0} d t+\sum_{k=1}^{d} \lambda_{\bullet}^{k} d Y_{t}^{k}
$$


This finishes the proof.

What the above theorem shows is that the projection residuals are greatly simplified if we make use of the functions $\left\{h^{1}, \cdots, h^{d}, \frac{1}{2}|h|^{2}\right\}$ in the definition of the exponential manifold, i.e. if we choose the functions $\left\{c_{1}, \cdots, c_{m}\right\}$ in such a way that the functions $\left\{h^{1}, \cdots, h^{d}, \frac{1}{2}|h|^{2}\right\}$ are in $\operatorname{span}\left\{c_{1}, \cdots, c_{m}\right\}$. Indeed, $\mathcal{R}_{t}^{k}\left(\sqrt{p_{t}^{\pi}}\right)=0$ for all $t \geq 0$, and all $k=0,1, \cdots, k$, whereas

$$
\frac{1}{\sqrt{p_{t}^{\pi}}} \mathcal{R}_{t}^{\bullet}\left(\sqrt{p_{t}^{\pi}}\right)=\frac{1}{2} \frac{\mathcal{L}_{t}^{*} p_{t}^{\pi}}{p_{t}^{\pi}}-\frac{1}{2}\left[c-\eta\left(\theta_{t}\right)\right]^{T}\left[g\left(\theta_{t}\right)\right]^{-1} E_{p\left(\cdot, \theta_{t}\right)}\left\{\mathcal{L}_{t} c\right\} .
$$

The diffusion coefficient in the stochastic differential equation (30) for the parameters $\theta_{t}$ is constant. This implies that (30) can be seen as either an Itô or a MFS stochastic differential equation, so that it satisfies the formal rules of calculus. Moreover, for the numerical solution of such an equation, the simpler Euler scheme coincides with the Milshtein scheme, which is a strongly convergent scheme of order 1 , see [12].

Notice also that we have still some freedom left, and we may wonder whether one can use this to select $m$ and the remaining functions $\left\{c_{s+1}, \cdots, c_{m}\right\}$ in order to reduce the total residual norm $r_{t}^{*}=r_{t}^{\bullet}$. However, a great prudence is needed, because the filter may become complicated and numerical problems may arise. See examples on the cubic sensor in Section 8. In general, a trade-off is necessary in order to obtain an accurate, but still not too involved, exponential family and the associated projection filter.

Similarly to the Theorem 6.1 above, we have the following

Theorem 6.2 Let $s:=\operatorname{rank}\left\{h^{1}, \cdots, h^{d}\right\} \leq d$. There exist $s$ linearly independent functions $\left\{c_{1}, \cdots, c_{s}\right\}$ defined on $\mathbf{R}^{n}$, such that for all $x \in \mathbf{R}^{n}$

$$
h^{k}(x)=\sum_{i=1}^{s} \lambda_{i}^{k} c_{i}(x)
$$

for $k=1, \cdots, d$. Remaining functions $\left\{c_{s+1}, \cdots, c_{m}\right\}$ are chosen such that

$$
S_{*}:=\{p(\cdot, \theta), \theta \in \Theta\}, \quad p(x, \theta):=\exp \left[\theta^{T} c(x)-\psi(\theta)\right],
$$

where $\Theta \subseteq \mathbf{R}^{m}$ is open, is an exponential family of probability densities.

Assume that, in addition to (A), (B) and (C), the coefficients $f_{t}$ and $a_{t}$ of the system (7), and the coefficients $c$ of the exponential family $S_{*}$ are such that:

$$
E_{p(\cdot, \theta)}\left\{\left|\alpha_{t, \theta}\right|^{2}\right\}<\infty,
$$

holds for all $\theta \in \Theta$, and all $t \geq 0$, where the expression of $\alpha_{t, \theta}$ is given in (16).

Then, for the projection filter associated with the exponential family $S_{*}$, the correction residual norms $r_{t}^{k}$ are identically zero for all $t \geq 0$, and all $k=1, \cdots, d$, and the stochastic differential equation for the parameters reduces to:

$$
d \theta_{t}=\left[g\left(\theta_{t}\right)\right]^{-1} E_{p\left(\cdot, \theta_{t}\right)}\left\{\mathcal{L}_{t} c\right\} d t-\left[g\left(\theta_{t}\right)\right]^{-1} E_{p\left(\cdot, \theta_{t}\right)}\left\{\frac{1}{2}|h|^{2}\left[c-\eta\left(\theta_{t}\right)\right]\right\} d t+\sum_{k=1}^{d} \lambda_{*}^{k} d Y_{t}^{k}
$$


where for all $k=1, \cdots, d$ the $m$-dimensional vector $\lambda_{*}^{k}$ is defined by

$$
\lambda_{*}^{k}=\left[\begin{array}{c}
\lambda_{1}^{k} \\
\vdots \\
\lambda_{s}^{k} \\
0 \\
\vdots \\
0
\end{array}\right]
$$

Under the assumptions on the coefficients, this equation has a unique solution, up to the a.s. positive exit time $\tau:=\inf \left\{t>0: \theta_{t} \notin \Theta\right\}$.

The proof is analogous to the proof of Theorem 6.1 , and is therefore omitted.

In this case, $\mathcal{R}_{t}^{k}\left(\sqrt{p_{t}^{\pi}}\right)=0$ for all $t \geq 0$, and all $k=1, \cdots, d$, whereas

$$
\frac{1}{\sqrt{p_{t}^{\pi}}} \mathcal{R}_{t}^{\bullet}\left(\sqrt{p_{t}^{\pi}}\right)=\frac{1}{2} \frac{\mathcal{L}_{t}^{*} p_{t}^{\pi}}{p_{t}^{\pi}}-\frac{1}{2}\left[c-\eta\left(\theta_{t}\right)\right]^{T}\left[g\left(\theta_{t}\right)\right]^{-1} E_{p\left(\cdot, \theta_{t}\right)}\left\{\mathcal{L}_{t} c\right\}
$$

and

$$
\begin{aligned}
\frac{1}{\sqrt{p_{t}^{\pi}}} \mathcal{R}_{t}^{0}\left(\sqrt{p_{t}^{\pi}}\right)= & \frac{1}{4}\left[|h|^{2}-E_{p\left(\cdot, \theta_{t}\right)}\left\{|h|^{2}\right\}\right] \\
& -\frac{1}{2}\left[c-\eta\left(\theta_{t}\right)\right]^{T}\left[g\left(\theta_{t}\right)\right]^{-1} E_{p\left(\cdot, \theta_{t}\right)}\left\{\frac{1}{2}|h|^{2}\left[c-\eta\left(\theta_{t}\right)\right]\right\} .
\end{aligned}
$$

\subsection{The case of discrete-time observations}

We conclude this section by presenting the effect of choosing the exponential family $S_{\text {. }}$, in the case of a nonlinear filtering problem with discrete-time observations. In this model, the state process is as in equation ( 7 ), i.e.

$$
d X_{t}=f_{t}\left(X_{t}\right) d t+\sigma_{t}\left(X_{t}\right) d W_{t},
$$

but only discrete-time observations are available

$$
z_{n}=h\left(X_{t_{n}}\right)+v_{n},
$$

at times $0=t_{0}<t_{1}<\cdots<t_{n}<\cdots$, where $\left\{v_{n}, n \geq 0\right\}$ is a Gaussian white noise sequence independent of $\left\{X_{t}, t \geq 0\right\}$.

The nonlinear filtering problem consists in finding the conditional density $p_{n}(x)$ of the state $X_{t_{n}}$ given the observations up to time $t_{n}$, i.e. such that $P\left[X_{t_{n}} \in d x \mid \mathcal{Z}_{n}\right]=p_{n}(x) d x$, where $\mathcal{Z}_{n}:=\sigma\left(z_{0}, \cdots, z_{n}\right)$. We define also the prediction conditional density $p_{n}^{-}(x) d x=$ $P\left[X_{t_{n}} \in d x \mid \mathcal{Z}_{n-1}\right]$. The sequence $\left\{p_{n}, n \geq 0\right\}$ satisfies a recurrent equation, and the transition from $p_{n-1}$ to $p_{n}$ is decomposed in two steps, as explained in [10], [16] : 
Prediction step Between time $t_{n-1}$ and $t_{n}$, we solve the Fokker-Planck equation

$$
\frac{\partial p_{t}^{n}}{\partial t}=\mathcal{L}_{t}^{*} p_{t}^{n}, \quad p_{t_{n-1}}^{n}=p_{n-1} .
$$

The solution at final time $t_{n}$ defines the prediction conditional density $p_{n}^{-}=p_{t_{n}}^{n}$.

Correction step At time $t_{n}$, the observation $z_{n}$ is combined with the prediction conditional density $p_{n}^{-}$via the Bayes rule

$$
p_{n}(x)=c_{n} \Psi_{n}(x) p_{n}^{-}(x),
$$

where $c_{n}$ is a normalizing constant, and $\Psi_{n}(x)$ denotes the likelihood function for the estimation of $X_{t_{n}}$ based on the observation $z_{n}$ only, i.e.

$$
\Psi_{n}(x):=\exp \left\{-\frac{1}{2}\left|z_{n}-h(x)\right|^{2}\right\} .
$$

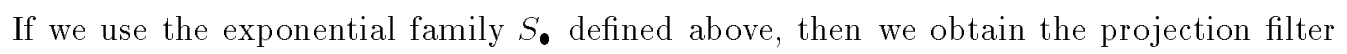
density $p\left(\cdot, \theta_{n}\right)$, and the transition from $\theta_{n-1}$ to $\theta_{n}$ is also decomposed in two steps :

Prediction step Between time $t_{n-1}$ and $t_{n}$, we solve the ODE

$$
g\left(\theta_{t}^{n}\right) \dot{\theta}_{t}^{n}=E_{p\left(\cdot, \theta_{t}^{n}\right)}\left\{\mathcal{L}_{t} c\right\}, \quad \theta_{t_{n-1}}^{n}=\theta_{n-1} .
$$

The solution at final time $t_{n}$ defines the prediction parameters $\theta_{n}^{-}=\theta_{t_{n}}^{n}$.

Correction step Substituting the approximation $p\left(\cdot, \theta_{n}^{-}\right)$into formula (35), we observe that the resulting density does not leave the exponential family $S_{\mathbf{0}}$. Indeed, it follows from (29) and (36) that

$$
\begin{aligned}
\Psi_{n}(x) & =\exp \left\{-\frac{1}{2}|h(x)|^{2}+\sum_{k=1}^{d} h^{k}(x) z_{n}^{k}-\frac{1}{2}\left|z_{n}\right|^{2}\right\} \\
& =\exp \left\{-\sum_{l=1}^{s} \lambda_{l}^{0} c_{l}(x)+\sum_{l=1}^{s}\left[\sum_{k=1}^{d} \lambda_{l}^{k} z_{n}^{k}\right] c_{l}(x)-\frac{1}{2}\left|z_{n}\right|^{2}\right\},
\end{aligned}
$$

and the parameters are updated according to the formula

$$
\theta_{n}=\theta_{n}^{-}-\lambda_{\bullet}^{0}+\sum_{k=1}^{d} \lambda_{\bullet}^{k} z_{n}^{k},
$$

which is exact.

RR ${ }^{\circ} 2598$ 


\section{An example of Bayesian system identification using the Gaussian projection filter}

In this section we consider a simple example of system identification and we derive explicit formulae for the projection filter applied to this problem. Consider the linear scalar system

$$
\begin{gathered}
d X_{t}=\mu X_{t} d t+d W_{t} \\
d Y_{t}=X_{t} d t+d V_{t},
\end{gathered}
$$

with the usual assumptions on the Brownian motions $\left\{W_{t}, t \geq 0\right\}$ and $\left\{V_{t}, t \geq 0\right\}$. The parameter $\mu$ is unknown. In order to estimate $\mu$, we introduce the constant stochastic process $\left\{U_{t}, t \geq 0\right\}$, which satisfies the following SDE

$$
d U_{t}=0
$$

so that by defining the augmented state $\left(X_{t}, U_{t}\right)$ the original system may be rewritten as

$$
\begin{aligned}
d\left[\begin{array}{l}
X_{t} \\
U_{t}
\end{array}\right] & =\left[\begin{array}{c}
U_{t} X_{t} \\
0
\end{array}\right] d t+\left[\begin{array}{l}
1 \\
0
\end{array}\right] d W_{t} \\
d Y_{t} & =\left[\begin{array}{ll}
1 & 0
\end{array}\right]\left[\begin{array}{l}
X_{t} \\
U_{t}
\end{array}\right] d t+d V_{t} .
\end{aligned}
$$

This is clearly a nonlinear system, with a bilinear state equation. As for the definition of the projection filter, we choose the manifold $S$ according to Theorem 6.1, i.e.

$S=S_{\bullet}=\{p(\cdot, \theta), \theta \in \Theta\}, \quad p(x, u, \theta)=\exp \left[\theta_{1} x+\theta_{2} x^{2}+\theta_{3} u+\theta_{4} u^{2}+\theta_{5} u x-\psi(\theta)\right]$,

where $\Theta \subset \mathbf{R}^{5}$ is open, $\theta_{2}<0, \theta_{4}<0$, and $\frac{1}{4} \theta_{5}^{2}<\theta_{2} \theta_{4}$ for all $\theta \in \Theta$. This is a Gaussian exponential family, and we choose the initial density as a Gaussian density with diagonal covariance matrix, since $U_{0}$ and $X_{0}$ are mutually independent. This covariance matrix is chosen very large so as to ensure that we are not assuming too much about the unknown parameter $\mu$. Let us develop now the procedure leading to the projection filter. We notice that $h(x, u)=x$ and $\frac{1}{2}|h(x, u)|^{2}=\frac{1}{2} x^{2}$, hence

$$
\lambda_{\bullet}^{0}=\left[\begin{array}{c}
0 \\
\frac{1}{2} \\
0 \\
0 \\
0
\end{array}\right], \quad \lambda_{\bullet}:=\lambda_{\bullet}^{1}=\left[\begin{array}{l}
1 \\
0 \\
0 \\
0 \\
0
\end{array}\right] .
$$

On the other hand

$$
\mathcal{L}=\frac{1}{2} \frac{\partial^{2}}{\partial x^{2}}+u x \frac{\partial}{\partial x}
$$


hence

$$
\begin{array}{ll}
\mathcal{L} c_{1}(x, u)=u x=c_{5}(x, u), & \mathcal{L} c_{2}(x, u)=1+2 u x^{2}, \\
\mathcal{L} c_{3}(x, u)=\mathcal{L} c_{4}(x, u)=0, & \mathcal{L} c_{5}(x, u)=u^{2} x .
\end{array}
$$

Notice that, using Lemma 2.3

$$
\begin{aligned}
E_{p(\cdot, \theta)}\left\{u x^{2}\right\} & =E_{p(\cdot, \theta)}\left\{c_{2} c_{3}\right\}=g_{23}(\theta)+\eta_{2}(\theta) \eta_{3}(\theta) \\
& =E_{p(\cdot, \theta)}\left\{c_{1} c_{5}\right\}=g_{15}(\theta)+\eta_{1}(\theta) \eta_{5}(\theta)
\end{aligned}
$$

and

$$
\begin{aligned}
E_{p(\cdot, \theta)}\left\{u^{2} x\right\} & =E_{p(\cdot, \theta)}\left\{c_{1} c_{4}\right\}=g_{14}(\theta)+\eta_{1}(\theta) \eta_{4}(\theta) \\
& =E_{p(\cdot, \theta)}\left\{c_{3} c_{5}\right\}=g_{35}(\theta)+\eta_{3}(\theta) \eta_{5}(\theta) .
\end{aligned}
$$

Therefore

$$
\gamma_{\bullet}(\theta):=E_{p(\cdot, \theta)}\{\mathcal{L} c\}=\left[\begin{array}{c}
\eta_{5}(\theta) \\
1+2\left[g_{23}(\theta)+\eta_{2}(\theta) \eta_{3}(\theta)\right] \\
0 \\
0 \\
g_{14}(\theta)+\eta_{1}(\theta) \eta_{4}(\theta)
\end{array}\right]
$$

Moreover, since we are in the Gaussian case, we can explicitly determine the normalizing constant $\psi(\theta)$

$$
\psi(\theta)=\log 2 \pi-\frac{1}{2} \log \chi(\theta)-\frac{1}{4 \theta_{2}}\left[\theta_{1}^{2}+\frac{\alpha(\theta)^{2}}{\chi(\theta)}\right]=\log 2 \pi-\frac{1}{2} \log \chi(\theta)-\frac{\beta(\theta)}{\chi(\theta)},
$$

where $\chi(\theta), \alpha(\theta)$ and $\beta(\theta)$ are defined as follows

$$
\begin{aligned}
& \chi(\theta):=4 \theta_{2} \theta_{4}-\theta_{5}^{2}, \\
& \alpha(\theta):=2 \theta_{2} \theta_{3}-\theta_{1} \theta_{5}, \\
& \beta(\theta):=\theta_{1}^{2} \theta_{4}+\theta_{2} \theta_{3}^{2}-\theta_{1} \theta_{3} \theta_{5} .
\end{aligned}
$$

Using Lemma 2.3 again, the expectation parameters $\eta_{1}(\theta), \cdots, \eta_{5}(\theta)$, and the entries of the Fisher information matrix $\left(g_{i j}(\theta)\right)$ are obtained as

$$
\eta_{i}(\theta)=\frac{\partial}{\partial \theta_{i}} \psi(\theta), \quad i=1, \cdots, 5
$$

RR $\mathrm{n}^{\circ} 2598$ 
and

$$
g_{i j}(\theta)=\frac{\partial^{2}}{\partial \theta_{i} \partial \theta_{j}} \psi(\theta), \quad i, j=1, \cdots, 5
$$

respectively.

The stochastic differential equation (30) for the parameters reduces to

$$
d \theta_{t}=\left[g\left(\theta_{t}\right)\right]^{-1} \gamma_{\bullet}\left(\theta_{t}\right) d t-\lambda_{\bullet}^{0} d t+\lambda_{\bullet} d Y_{t} .
$$

The equation (31) for the prediction residual reduces to

$$
\frac{1}{\sqrt{p_{t}^{\pi}}} \mathcal{R}_{t}^{\bullet}\left(\sqrt{p_{t}^{\pi}}\right)=\frac{1}{2} \frac{\mathcal{L}^{*} p_{t}^{\pi}}{p_{t}^{\pi}}-\frac{1}{2}\left[c-\eta\left(\theta_{t}\right)\right]^{T}\left[g\left(\theta_{t}\right)\right]^{-1} \gamma_{\bullet}\left(\theta_{t}\right)
$$

from which the total residual norm $r_{t}^{*}=r_{t}^{\bullet}$ can be easily computed.

This example illustrates how in some situations the residual and the filter equations may be computed exactly via stochastic differential equations involving rational functions of the canonical parameters $\theta$. Moreover, this example shows a situation in which it is natural to choose a Gaussian simplifying manifold $S_{\text {. }}$. The difference with the extended Kalman filter used as a parameter estimator is that in our case we have an explicit expression for the well defined quantity $r_{t}^{\bullet}$ representing the quality of the approximation at every time instant.

\section{Exponential projection filters for the cubic sensor}

We consider as an application of the exponential projection filter the explicit formula for the cubic sensor, see also [8]. We consider the scalar system

$$
\begin{aligned}
& d X_{t}=\sigma d W_{t} \\
& d Y_{t}=X_{t}^{3} d t+d V_{t}
\end{aligned}
$$

with the usual independence assumptions for the standard Brownian motions $\left\{W_{t}, t \geq 0\right\}$ and $\left\{V_{t}, t \geq 0\right\}$ and where $\sigma$ is a real constant. This system is interesting for several reasons. First, the simplicity of the state process. Secondly, the infinite dimensionality of the optimal filter for the cubic sensor ensures that we are really facing a problem of approximating an infinite dimensional filter by a finite dimensional one. The fact that the optimal filter for the cubic sensor is infinite dimensional was proven in [9].

Let us apply the projection filter to this system using different exponential families in order to illustrate how the filter depends on the manifold.

\subsection{The six dimensional exponential projection filter}

We choose the manifold $S$ according to Theorem 6.1, i.e.

$S=S_{\bullet}=\{p(\cdot, \theta), \theta \in \Theta\}, \quad p(x, \theta)=\exp \left[\theta_{1} x+\theta_{2} x^{2}+\theta_{3} x^{3}+\theta_{4} x^{4}+\theta_{5} x^{5}+\theta_{6} x^{6}-\psi(\theta)\right]$, 
where $\Theta$ is open in $\mathbf{R}^{6}$ and $\theta_{6}<0$, for all $\theta \in \Theta$.

We notice that $h(x)=x^{3}$ and $\frac{1}{2}|h(x)|^{2}=\frac{1}{2} x^{6}$, hence

$$
\lambda_{\bullet}^{0}=\left[\begin{array}{c}
0 \\
0 \\
0 \\
0 \\
0 \\
\frac{1}{2}
\end{array}\right], \quad \lambda_{\bullet}:=\lambda_{\bullet}^{1}=\left[\begin{array}{l}
0 \\
0 \\
1 \\
0 \\
0 \\
0
\end{array}\right] .
$$

On the other hand, $c_{j}(x)=x^{j}$, for $j=1, \cdots, 6$, hence

$$
\mathcal{L} c_{j}(x)=\frac{1}{2} \sigma^{2} \frac{\partial^{2} c_{j}(x)}{\partial x^{2}}= \begin{cases}\frac{1}{2} \sigma^{2} j(j-1) x^{j-2}, & \text { for } j=2, \cdots, 6 \\ 0, & \text { for } j=1\end{cases}
$$

and therefore

$$
E_{p(\cdot, \theta)}\left\{\mathcal{L} c_{j}\right\}= \begin{cases}\frac{1}{2} \sigma^{2} j(j-1) \eta_{j-2}(\theta), & \text { for } j=2, \cdots, 6 \\ 0, & \text { for } j=1\end{cases}
$$

which requires the evaluation of $\eta_{0}(\theta), \cdots, \eta_{4}(\theta)$. We define

$$
\gamma_{\bullet}(\theta):=\frac{1}{2} \sigma^{2}\left[\begin{array}{c}
0 \\
2 \eta_{0}(\theta) \\
6 \eta_{1}(\theta) \\
12 \eta_{2}(\theta) \\
20 \eta_{3}(\theta) \\
30 \eta_{4}(\theta)
\end{array}\right]=E_{p(\cdot, \theta)}\{\mathcal{L} c\}
$$

Finally, the entries of the Fisher information matrix $\left(g_{i j}(\theta)\right)$ are obtained according to $(6)$, i.e.

$$
g_{i j}(\theta)=\eta_{i+j}(\theta)-\eta_{i}(\theta) \eta_{j}(\theta), \quad i, j=1, \cdots, 6
$$

which requires the evaluation of $\eta_{1}(\theta), \cdots, \eta_{12}(\theta)$. However, $\eta_{0}(\theta)=1$ and it follows from Lemma 2.3 that only $\eta_{1}(\theta), \cdots, \eta_{5}(\theta)$ need to be evaluated, since $\eta_{6}(\theta), \cdots, \eta_{12}(\theta)$ can be obtained according to $(5)$.

The stochastic differential equation (30) for the parameters reduces to

$$
d \theta_{t}=\left[g\left(\theta_{t}\right)\right]^{-1} \gamma_{\bullet}\left(\theta_{t}\right) d t-\lambda_{\bullet}^{0} d t+\lambda_{\bullet} d Y_{t} .
$$

The equation (31) for the prediction residual reduces to

$$
\frac{1}{\sqrt{p_{t}^{\pi}}} \mathcal{R}_{t}^{\bullet}\left(\sqrt{p_{t}^{\pi}}\right)=\frac{1}{2} \frac{\mathcal{L}^{*} p_{t}^{\pi}}{p_{t}^{\pi}}-\frac{1}{2}\left[c-\eta\left(\theta_{t}\right)\right]^{T}\left[g\left(\theta_{t}\right)\right]^{-1} \gamma_{\bullet}\left(\theta_{t}\right),
$$

RR $n^{\circ} 2598$ 
from which the total residual norm $r_{t}^{*}=r_{t}^{\bullet}$ can be easily computed.

Finally, we indicate a quantity which can be used to estimate the state of the system at time $t$. It is well known that, if the conditional density $p_{t}$ is available, then the best (minimum variance) estimator of $X_{t}$ is the conditional expectation

$$
\widehat{X}_{t}:=E_{p_{t}}\{x\}=\int x p_{t}(x) d \lambda(x) .
$$

As we can rely only on the approximated density $p\left(\cdot, \theta_{t}\right)$, we shall consider, as an estimate of the state, the expectation w.r.t. this approximated density :

$$
\eta_{1}\left(\theta_{t}\right)=E_{p\left(\cdot, \theta_{t}\right)}\{x\}=\int x p\left(x, \theta_{t}\right) d \lambda(x) .
$$

\subsection{The four dimensional exponential projection filter}

In this section we choose the manifold $S$ according to Theorem 6.2, i.e.

$$
S=S_{*}=\{p(\cdot, \theta), \theta \in \Theta\}, \quad p(x, \theta)=\exp \left[\theta_{1} x+\theta_{2} x^{2}+\theta_{3} x^{3}+\theta_{4} x^{4}-\psi(\theta)\right],
$$

where $\Theta \subseteq \mathbf{R}^{4}$ is open and $\theta_{4}<0$, for all $\theta \in \Theta$.

We notice that $h(x)=x^{3}$, hence

$$
\lambda_{*}:=\lambda_{*}^{1}=\left[\begin{array}{l}
0 \\
0 \\
1 \\
0
\end{array}\right]
$$

On the other hand, $c_{j}(x)=x^{j}$, for $j=1, \cdots, 4$, hence

$$
\mathcal{L} c_{j}(x)=\frac{1}{2} \sigma^{2} \frac{\partial^{2} c_{j}(x)}{\partial x^{2}}= \begin{cases}\frac{1}{2} \sigma^{2} j(j-1) x^{j-2}, & \text { for } j=2, \cdots, 4 \\ 0, & \text { for } j=1\end{cases}
$$

and therefore

$$
E_{p(\cdot, \theta)}\left\{\mathcal{L} c_{j}\right\}= \begin{cases}\frac{1}{2} \sigma^{2} j(j-1) \eta_{j-2}(\theta), & \text { for } j=2, \cdots, 4 \\ 0, & \text { for } j=1\end{cases}
$$

which requires the evaluation of $\eta_{0}(\theta), \cdots, \eta_{2}(\theta)$. We define

$$
\gamma_{*}(\theta):=\frac{1}{2} \sigma^{2}\left[\begin{array}{c}
0 \\
2 \eta_{0}(\theta) \\
6 \eta_{1}(\theta) \\
12 \eta_{2}(\theta)
\end{array}\right]=E_{p(\cdot, \theta)}\{\mathcal{L} c\}
$$


Similarly, we notice that $\frac{1}{2}|h(x)|^{2}=\frac{1}{2} x^{6}$, hence

$$
\frac{1}{2}|h(x)|^{2}\left[c_{j}(x)-\eta_{j}(\theta)\right]=\frac{1}{2}\left[x^{6+j}-x^{6} \eta_{j}(\theta)\right], \quad j=1, \cdots, 4
$$

and

$$
E_{p(\cdot, \theta)}\left\{\frac{1}{2}|h|^{2}\left[c_{j}-\eta_{j}(\theta)\right]\right\}=\frac{1}{2}\left[\eta_{6+j}(\theta)-\eta_{6}(\theta) \eta_{j}(\theta)\right], \quad j=1, \cdots, 4
$$

which requires the evaluation of $\eta_{1}(\theta), \cdots, \eta_{4}(\theta)$ and $\eta_{6}(\theta), \cdots, \eta_{10}(\theta)$. We define

$$
\gamma_{*}^{0}(\theta):=\frac{1}{2}\left[\begin{array}{c}
\eta_{7}(\theta)-\eta_{6}(\theta) \eta_{1}(\theta) \\
\eta_{8}(\theta)-\eta_{6}(\theta) \eta_{2}(\theta) \\
\eta_{9}(\theta)-\eta_{6}(\theta) \eta_{3}(\theta) \\
\eta_{10}(\theta)-\eta_{6}(\theta) \eta_{4}(\theta)
\end{array}\right]=E_{p(\cdot, \theta)}\left\{\frac{1}{2}|h|^{2}[c-\eta(\theta)]\right\}
$$

Finally, the entries of the Fisher information matrix $\left(g_{i j}(\theta)\right)$ are obtained according to $(6)$, i.e.

$$
g_{i j}(\theta)=\eta_{i+j}(\theta)-\eta_{i}(\theta) \eta_{j}(\theta), \quad i, j=1, \cdots, 4
$$

which requires the evaluation of $\eta_{1}(\theta), \cdots, \eta_{8}(\theta)$. However, $\eta_{0}(\theta)=1$ and it follows from Lemma 2.3 that only $\eta_{1}(\theta), \cdots, \eta_{3}(\theta)$ need to be evaluated, since $\eta_{4}(\theta), \cdots, \eta_{10}(\theta)$ can be obtained according to $(5)$.

The stochastic differential equation (32) for the parameters reduces to

$$
d \theta_{t}=\left[g\left(\theta_{t}\right)\right]^{-1} \gamma_{*}\left(\theta_{t}\right) d t-\left[g\left(\theta_{t}\right)\right]^{-1} \gamma_{*}^{0}\left(\theta_{t}\right) d t+\lambda_{*} d Y_{t}
$$

The equations (33) and (34) for the prediction and correction residuals reduce to

$$
\frac{1}{\sqrt{p_{t}^{\pi}}} \mathcal{R}_{t}^{\bullet}\left(\sqrt{p_{t}^{\pi}}\right)=\frac{1}{2} \frac{\mathcal{L}^{*} p_{t}^{\pi}}{p_{t}^{\pi}}-\frac{1}{2}\left[c-\eta\left(\theta_{t}\right)\right]^{T}\left[g\left(\theta_{t}\right)\right]^{-1} \gamma_{*}\left(\theta_{t}\right),
$$

and

$$
\frac{1}{\sqrt{p_{t}^{\pi}}} \mathcal{R}_{t}^{0}\left(\sqrt{p_{t}^{\pi}}\right)=\frac{1}{4}\left[x^{6}-\eta_{6}\left(\theta_{t}\right)\right]-\frac{1}{2}\left[c-\eta\left(\theta_{t}\right)\right]^{T}\left[g\left(\theta_{t}\right)\right]^{-1} \gamma_{*}^{0}\left(\theta_{t}\right),
$$

respectively, from which the total residual norm $r_{t}^{*}$ can be easily computed.

Finally, as in Section 8.1 our approximation of the minimum variance estimate of the state at time $t$ is the first expectation parameter $\eta_{1}\left(\theta_{t}\right)$. We conclude by observing that the filter given in this section can be implemented via a numerical scheme involving numerical integration techniques. Such a scheme has been written as a Fortran program, yielding simulations that we describe in the next section.

\section{Numerical simulations for the cubic sensor}

In this section we present a numerical scheme which was used to implement the projection filter derived in Section 8.2, and we present also simulation results based on this numerical 
scheme. From the previous discussion, we need to compute the moments $\eta_{1}, \cdots, \eta_{10}$ up to order ten, but according to Lemma 2.3, these moments can be computed from the first three moments $\eta_{1}, \cdots, \eta_{3}$ only by using the recursion formula (5).

We applied a Euler scheme to solve the stochastic differential equation (39) numerically. Since the diffusion coefficient in this equation is constant, the Euler scheme coincides with the Milshtein scheme, and hence the error is of order $\Delta$, where $\Delta$ is the chosen time step. In general, if the diffusion coefficient would also depend on the state $\theta$ then the error would be of order $\sqrt{\Delta}$ only. For a detailed treatment of numerical methods for stochastic differential equations, see [12].

We outline the main steps of the algorithm :

(i) Let an initial $\theta_{0}$ be given. Choose a time step $\Delta$ and set $t=0$.

(ii) Assign $\theta:=\theta_{0}$.

(iii) Compute numerically the integral

$$
I(\theta):=\exp [\psi(\theta)]=\int_{-\infty}^{+\infty} \exp \left[\theta_{1} x+\theta_{2} x^{2}+\theta_{3} x^{3}+\theta_{4} x^{4}\right] d \lambda(x)
$$

(iv) Compute the three following integrals, so as to obtain the first three expectation parameters :

$$
\begin{aligned}
\eta_{i}(\theta) & =E_{p(\cdot, \theta)}\left\{x^{i}\right\} \\
& =\frac{1}{I(\theta)} \int_{-\infty}^{+\infty} x^{i} \exp \left[\theta_{1} x+\theta_{2} x^{2}+\theta_{3} x^{3}+\theta_{4} x^{4}\right] d \lambda(x), \quad i=1,2,3 .
\end{aligned}
$$

(v) Compute the higher order moments $\eta_{4}, \cdots, \eta_{10}$ via the algebraic recursion formula given in $(5)$.

(vi) Substitute the above quantities in equations (37) and (38), so as to obtain the coefficients $\gamma_{*}(\theta)$ and $\gamma_{*}^{0}(\theta)$ respectively.

(vii) Compute the Fisher information matrix

$$
g_{i j}(\theta)=E_{p(\cdot, \theta)}\left\{x^{i} x^{j}\right\}-\eta_{i} \eta_{j}=\eta_{i+j}-\eta_{i} \eta_{j}, \quad i, j=1, \cdots, 4
$$

(viii) Invert $\left(g_{i j}(\theta)\right)$ so as to obtain $\left(g^{i j}(\theta)\right)$.

(ix) Collect the new observation $Y_{t+\Delta}$ at time $t+\Delta$ (here a discretization scheme is needed), and let $\Delta Y=Y_{t+\Delta}-Y_{t}$.

(x) Compute the approximate variation $\Delta \theta$ of the canonical parameters between times $t$ and $t+\Delta$, according to the simple Euler scheme

$$
\Delta \theta=[g(\theta)]^{-1} \gamma_{*}(\theta) \Delta-[g(\theta)]^{-1} \gamma_{*}^{0}(\theta) \Delta+\lambda_{*} \Delta Y .
$$


(xi) Assign $\theta:=\theta+\Delta \theta$ and $t:=t+\Delta$.

(xii) Start again from point (iii).

As noticed in step (v), all we need is to compute the integrals

$$
\int_{-\infty}^{+\infty} x^{i} \exp \left[\theta_{1} x+\theta_{2} x^{2}+\theta_{3} x^{3}+\theta_{4} x^{4}\right] d \lambda(x), \quad i=0, \cdots, 3 .
$$

We used routines from the scientific library NAG for this purpose.

Once a numerical approximation of the projection filter parameters $\theta_{t}$ has been computed, we can compare the corresponding density $p_{t}^{\pi}=p\left(\cdot, \theta_{t}\right)$ to the solution $p_{t}$ of the Kushner-Stratonovich equation, i.e. to the optimal filter density. Actually, a numerical approximation of $p_{t}$ was used, based on a discretization of the state space with approximately 400 grid points, and on numerical techniques for the solution of stochastic differential equations, see [12] and [4].

The comparison between numerical approximations of the densities $p_{t}^{\pi}$ and $p_{t}$ can be done qualitatively, based on graphical outputs, or we can compute (a numerical approximation of) some distance, such as the Kullback-Leibler information

$$
K\left(p_{t}, p_{t}^{\pi}\right):=\int \log \frac{p_{t}(x)}{p_{t}^{\pi}(x)} p_{t}(x) d \lambda(x),
$$

the Hellinger distance

$$
d\left(p_{t}, p_{t}^{\pi}\right):=\int\left(\sqrt{p_{t}^{\pi}(x)}-\sqrt{p_{t}(x)}\right)^{2} d \lambda(x)=2\left[1-\int \sqrt{p_{t}^{\pi}(x)} \sqrt{p_{t}(x)} d \lambda(x)\right],
$$

etc. We can also compute an approximation of the total residual norm

$$
r_{t}^{*}:=\left\|\mathcal{R}_{t}^{\bullet}\left(\sqrt{p_{t}^{\pi}}\right)-\mathcal{R}_{t}^{0}\left(\sqrt{p_{t}^{\pi}}\right)\right\|,
$$

which depends only on the projection filter density. As remarked in Section 8.2, the remaining correction residual norms $r_{t}^{k}$ vanish for all $t \geq 0$ and all $k=1, \cdots, d$. Moreover, to compute the total residual norm $r_{t}^{*}$ we still need to evaluate only the first three moments.

We begin with some general remarks about our simulation results. These results show that the projection filter density is usually very close to the optimal filter density, when the latter is not too sharp (i.e. not too close to a Dirac mass). What would be missing in a Gaussian assumed density filter or in an extended Kalman filter is the possibility to allow bimodality in the filter density. As the fourth degree exponential family allows such bimodality, in principle the optimal filter density could be approximated at least qualitatively by a density in this family. This was actually observed in our simulations.

Moreover, we can have an a posteriori indication of the accuracy of the projection filter from the graphical representation of the total residual norm as a function of time. Indeed, there are time instants where the optimal filter density and the projection filter density are 
quite different, but these are exactly the time instants where the total residual norm exhibits large values. An additional observation that we could make on our simulations is that after a reasonably small time the total residual norm returns towards zero, and correspondingly the projection filter density is again very close to the optimal filter density. To summarize, there are some differences, but they are limited in time, and do not seem to affect the global behaviour of the projection filter.

On time intervals where the true state is far from the singular point $x=0$ of the observation function $h(x)=x^{3}$, experience shows that the smaller the observation noise, the sharper and higher are the peaks of the total residual norm. Notice first that if the observation noise is small, then on such time intervals the optimal filter densities are concentrated around the true state trajectory, i.e. are tracking accurately a very irregular trajectory. As a result, the difference between the mean value of the optimal filter density and the mean value of the projection filter density has to be really small, i.e. smaller than the variance of the optimal filter density, to guarantee that the Hellinger distance between the optimal filter density and the projection filter density is not too large. This is reflected in the fast dynamics of both the Kushner-Stratonovich equation, and the equation for the projection filter parameters, and makes the numerical implementation of the projection filter difficult when the observation noise is small.

In the following we discuss the simulations in detail, and we present some graphical outputs which illustrate our general remarks. In the two scalar examples below, the variance $R$ of the observation noise does not satisfy $R=1$. However, the formulas given in the paper could easily be adapted to this more general situation.

Example 1: We present here a first simulation of the fourth degree exponential projection filter based on the following data :

\begin{tabular}{||c|c||}
\hline $\begin{array}{c}\text { (unnormalized) initial density } \\
\text { variance } Q \text { of the state noise }\end{array}$ & $\exp \left[-\frac{1}{2} x^{2}-\frac{1}{4} x^{4}\right]$ \\
variance $R$ of the observation noise \\
time step $\Delta$ \\
final time
\end{tabular}

In this first example we are mainly concerned in showing that our choice of the fourth degree exponential family is well chosen. Visualizing the time evolution of both the optimal filter density and the projection filter density was made possible with the software ZPB developed at INRIA. We observed that qualitatively the projection filter was good, as the two densities had roughly the same shape at every time instant. In this paper we display the two densities at three time instants. We start by Figures 1 and 2 which show the true state and the estimate (mean value) provided by the projection filter density respectively, as functions of time. This estimate is not accurate because on this simulation the true state 
stays most of the time around the singular point $x=0$ of the observation function. Indeed, Figures 3 and 4 show that the mean value of the optimal filter density does not provide an accurate estimate of the true state either. We are also interested in comparing the projection filter with the optimal filter, and not only with the true state. In this respect, Figures 5 and 7 , show that the two filter estimates agree surprisingly well. Notice also the behaviour of the total residual norm in Figures 6 and 8 : the time instants where the two filter estimates are significantly different are characterized by large peaks in the total residual norm. This kind of simulation, where the conditional density is concentrated around the singular point of the observation function, is important because it is in such situations that Gaussian assumed density filters and extended Kalman filters would usually fail. The shape of the density is quickly varying, becoming often bimodal and asymmetric, so that a Gaussian family is definitely not a good choice to base a finite dimensional filtering on. We make this evident by displaying the optimal filter and the projection filter densities at different time instants, in Figures 9, 10, 11, 12, 13, and 14.

Example 2 : The second example is based on the following data :

\begin{tabular}{||c|c||}
\hline $\begin{array}{c}\text { (unnormalized) initial density } \\
\text { variance } Q \text { of the state noise }\end{array}$ & $\exp \left[-\frac{1}{2}\left(x-\frac{3}{4}\right)^{2}-\frac{1}{4}\left(x-\frac{3}{4}\right)^{4}\right]$ \\
variance $R$ of the observation noise & 1 \\
time step $\Delta$ & 9 \\
final time & 0.005 \\
\end{tabular}

We begin by comparing the true state with the estimate (mean value) provided by the projection filter density. This is illustrated in Figures 15 and 16. It is clear from this graphical output that the state is not estimated accurately, and this is due to the fact that we have a large observation noise. Anyway, this is the case also for the optimal filter, as we can see in Figures 17 and 18. Nonetheless, our main concern is in the comparison between the projection filter and the optimal filter. This comparison is provided by Figures 19 and 21. The projection filter and the optimal filter estimates agree surprisingly well, and the time instants where they are significantly different are characterized by peaks of the total residual norm, which is shown in Figures 20 and 22.

$\operatorname{RR} \mathrm{n}^{\circ} 2598$ 


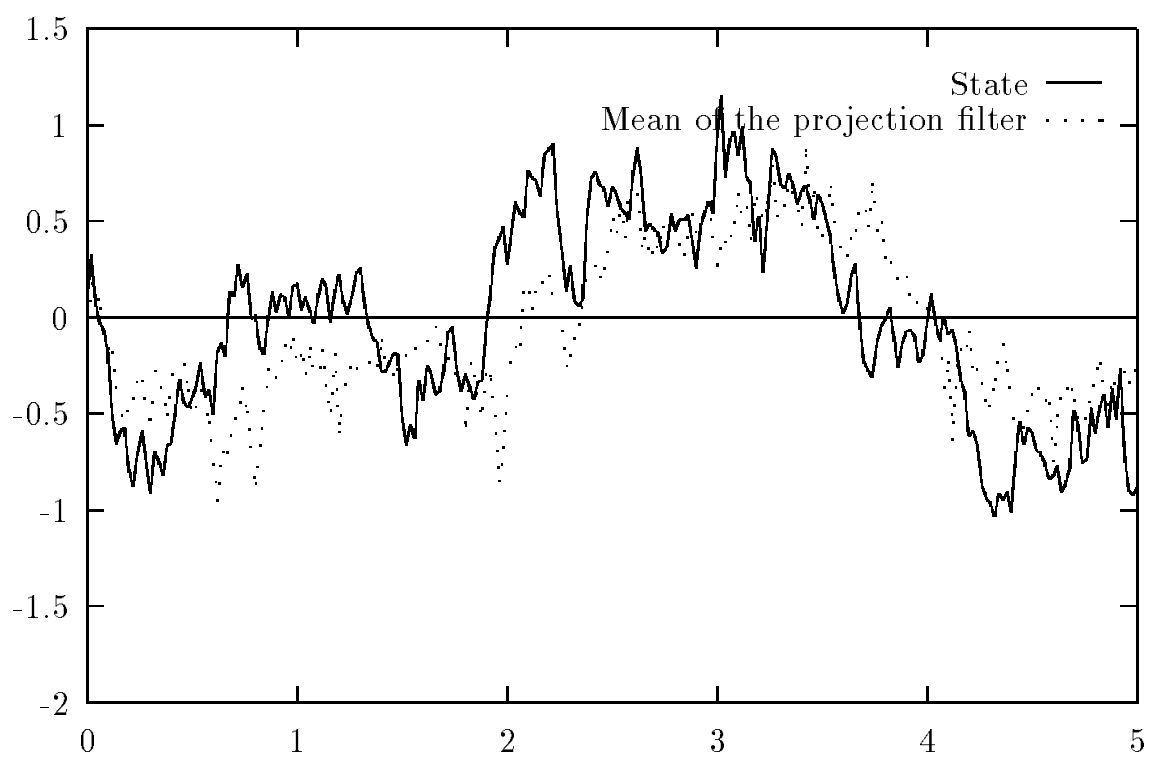

Figure 1: True state and mean from the projection filter between 0 and 5

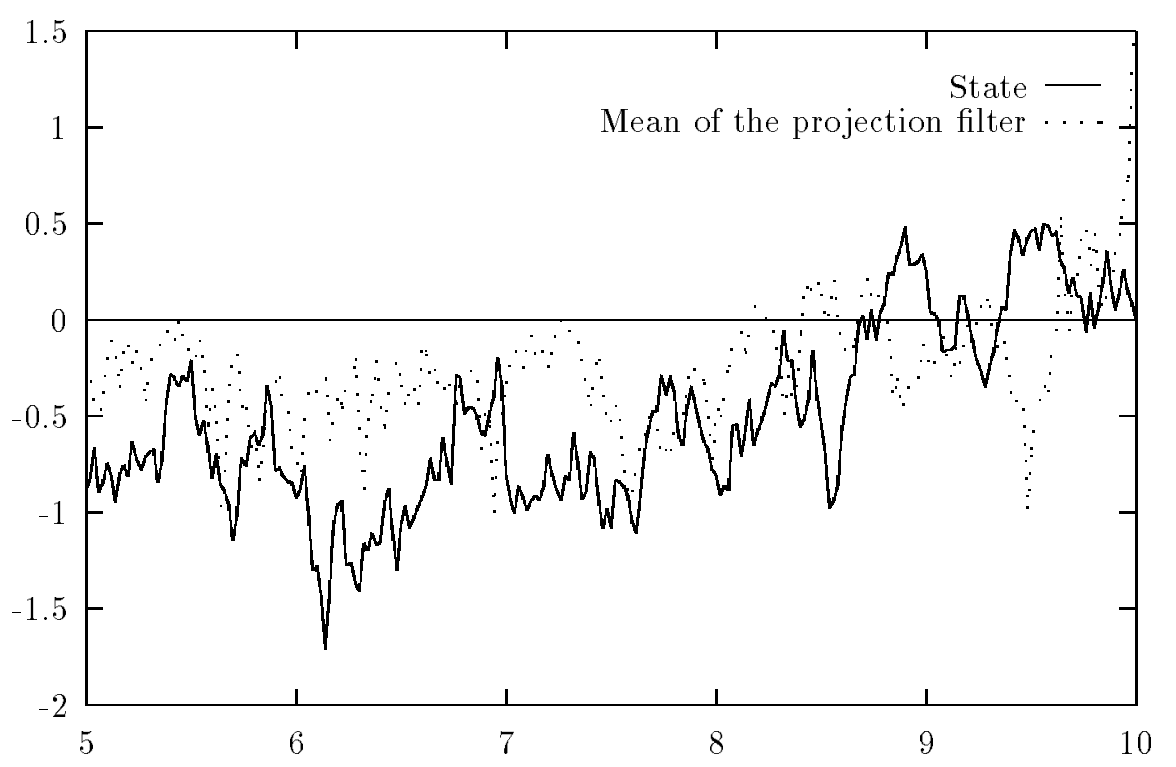

Figure 2: True state and mean from the projection filter between 5 and 10 


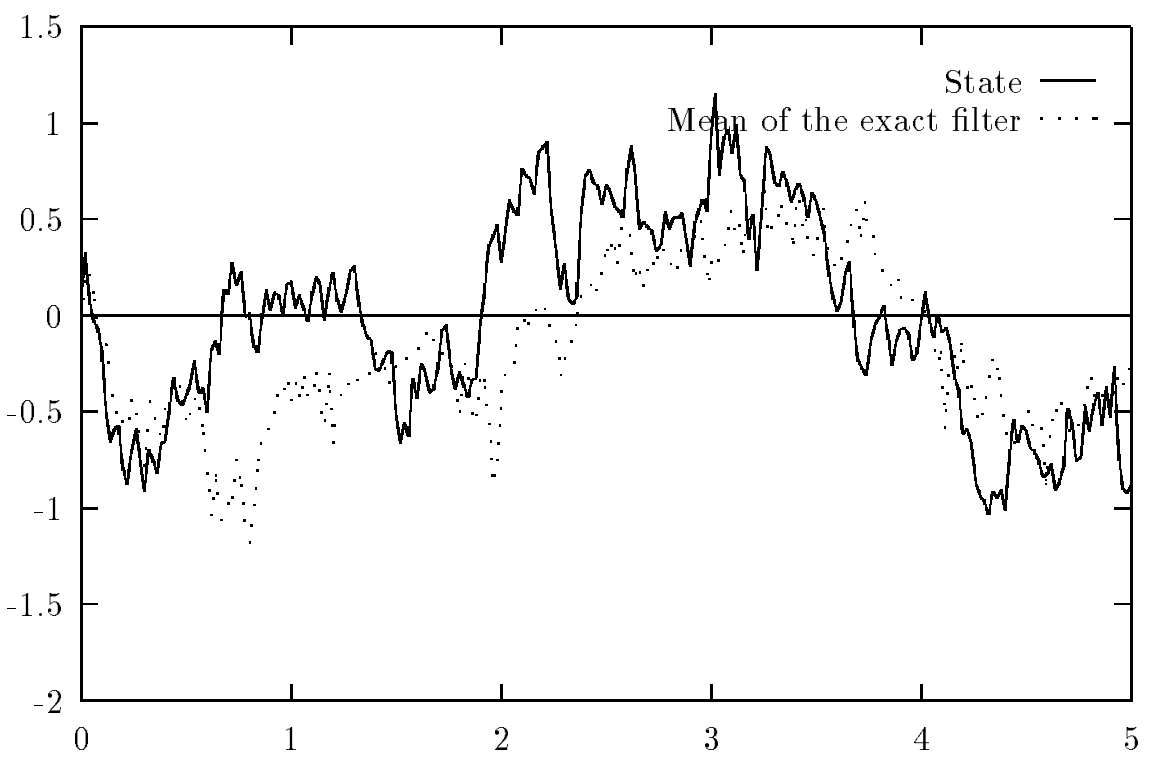

Figure 3: True state and mean from the optimal filter between 0 and 5

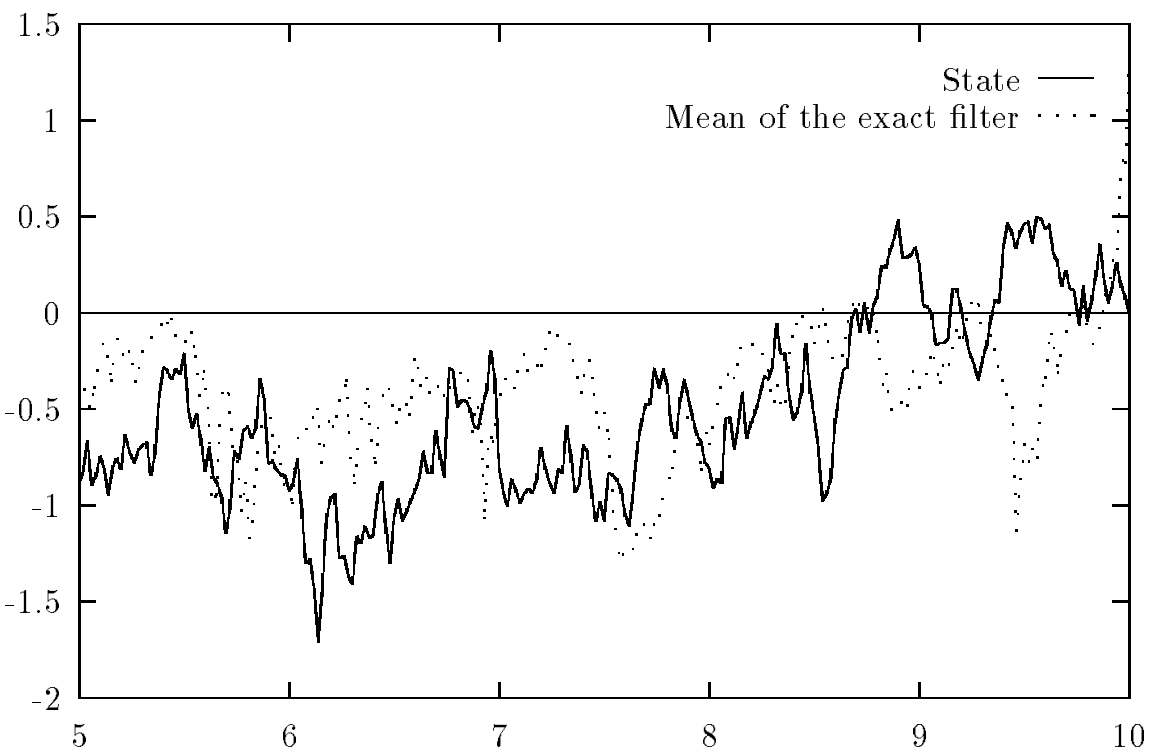

Figure 4: True state and mean from the optimal filter between 5 and 10 


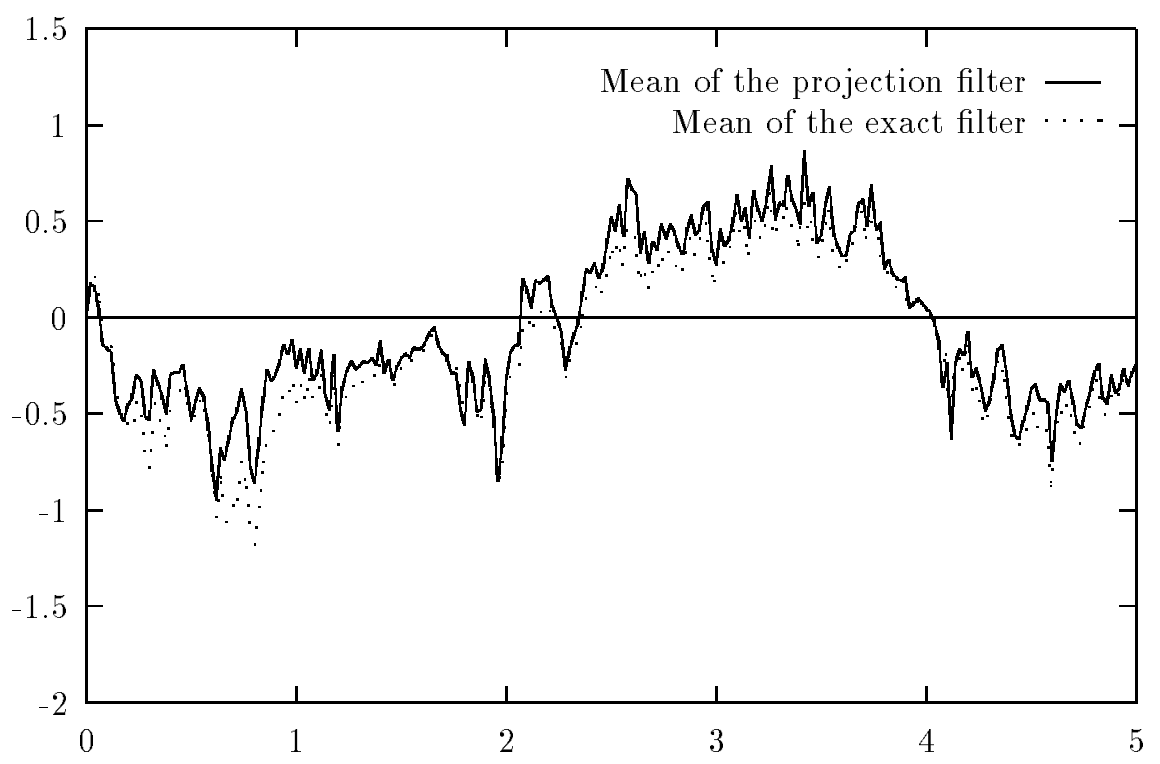

Figure 5: Mean from the projection filter and from the optimal filter between 0 and 5

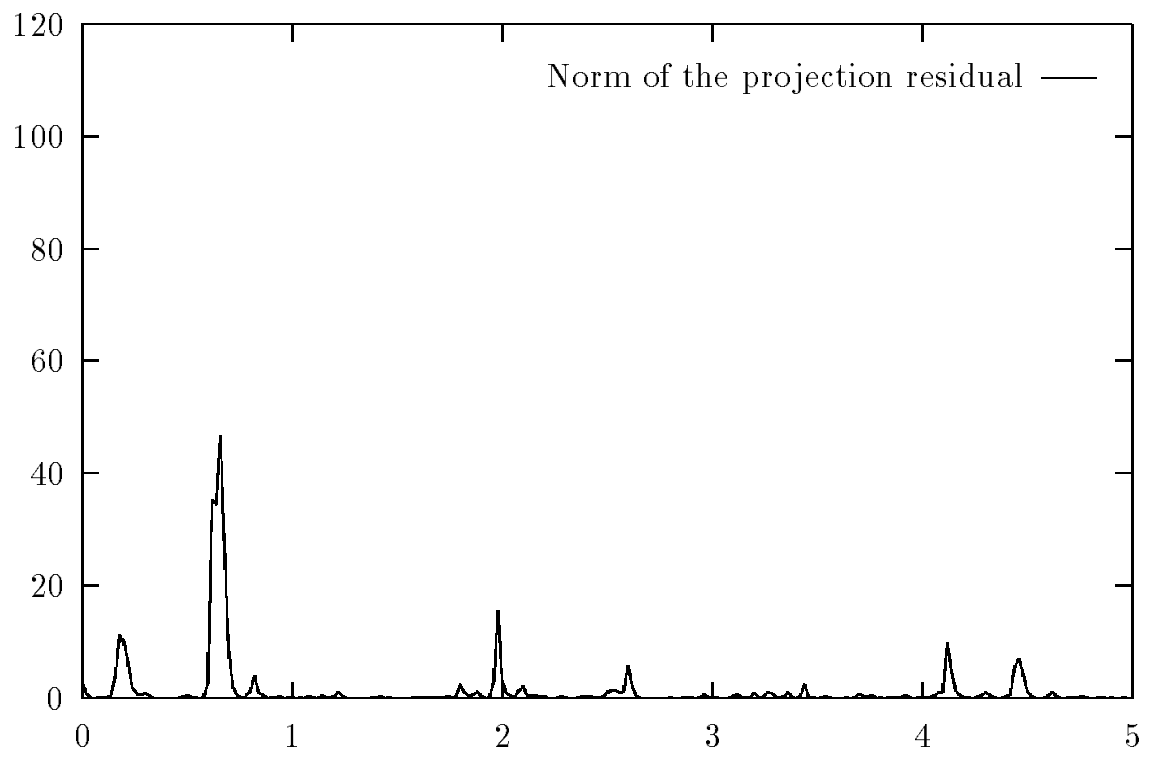

Figure 6: Projection residual between 0 and 5 


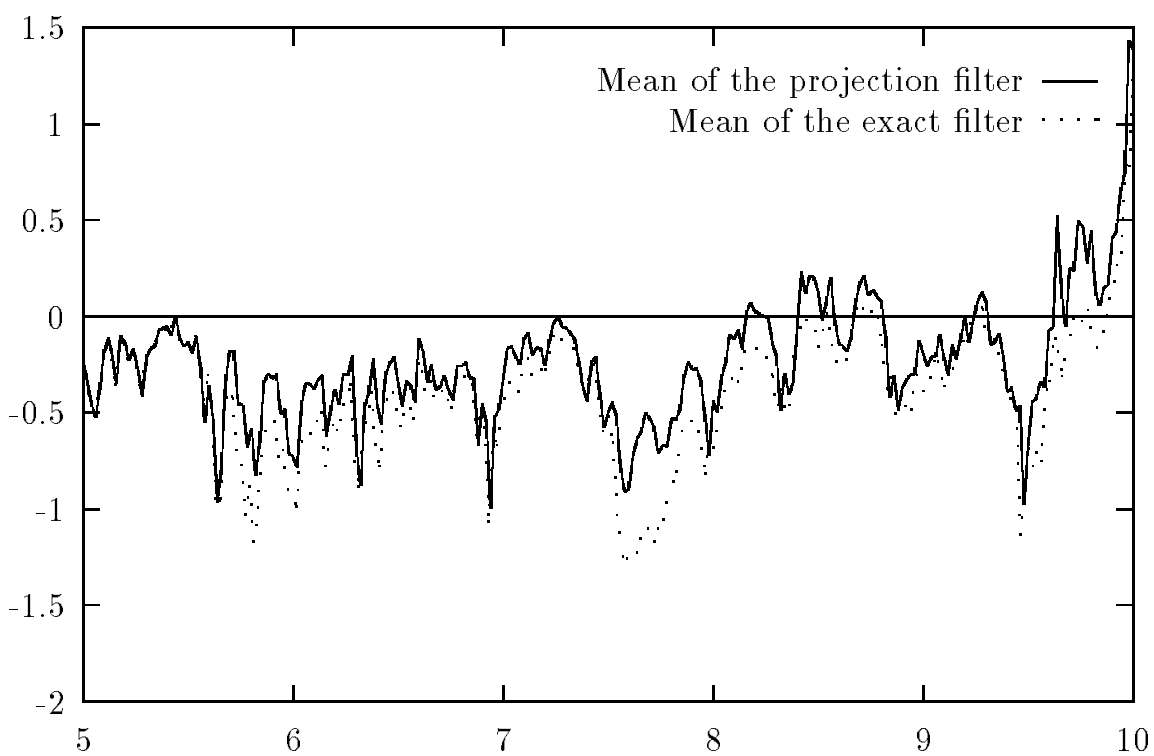

Figure 7: Mean from the projection filter and from the optimal filter between 5 and 10

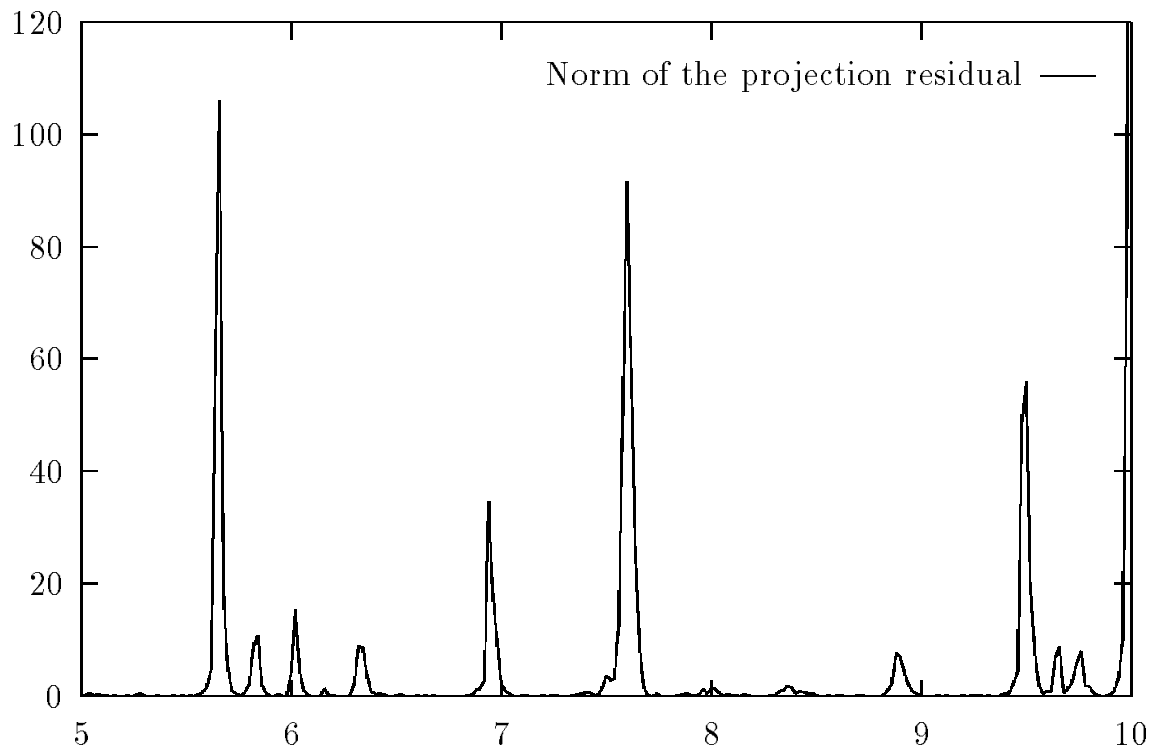

Figure 8: Projection residual between 5 and 10 


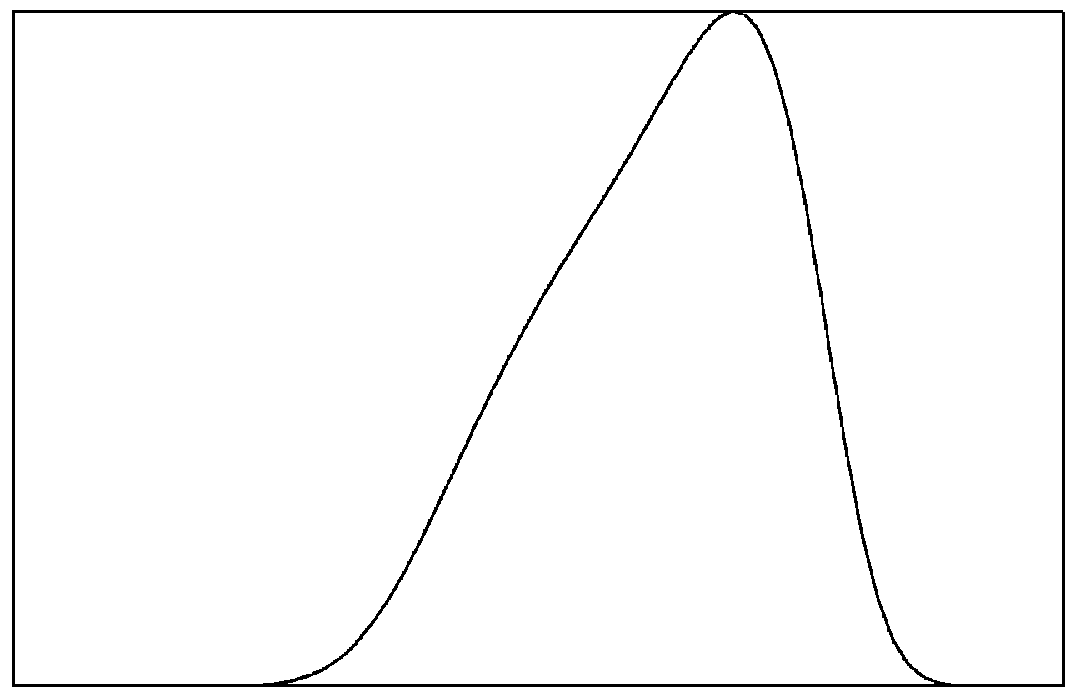

Figure 9: Optimal filter density at 3.70

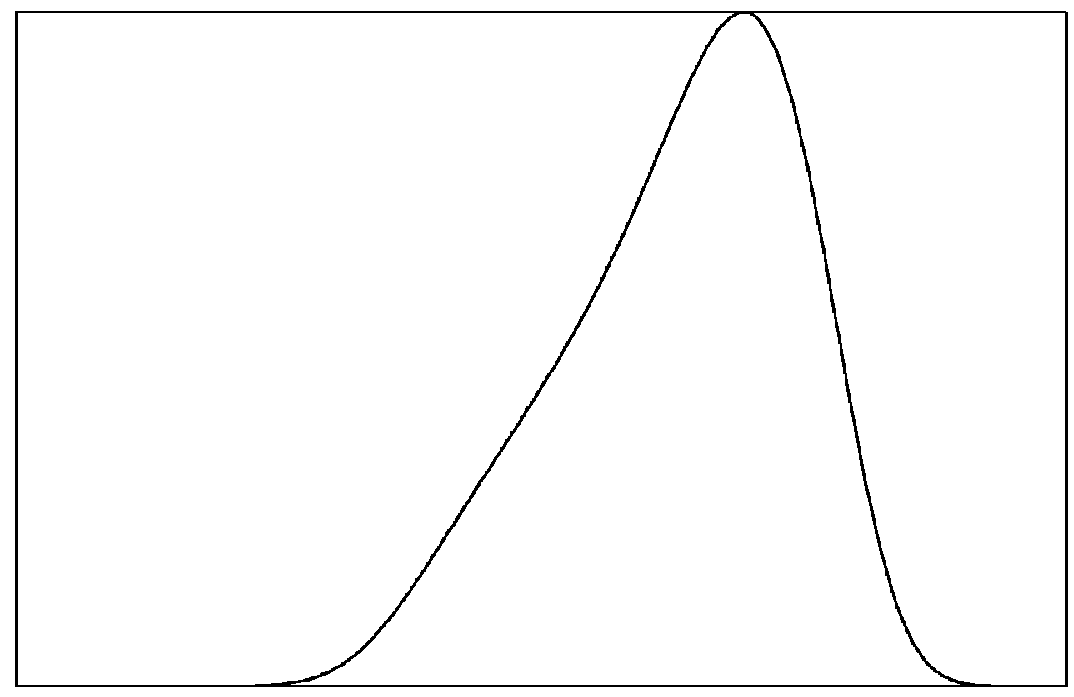

Figure 10: Projection filter density at 3.70 


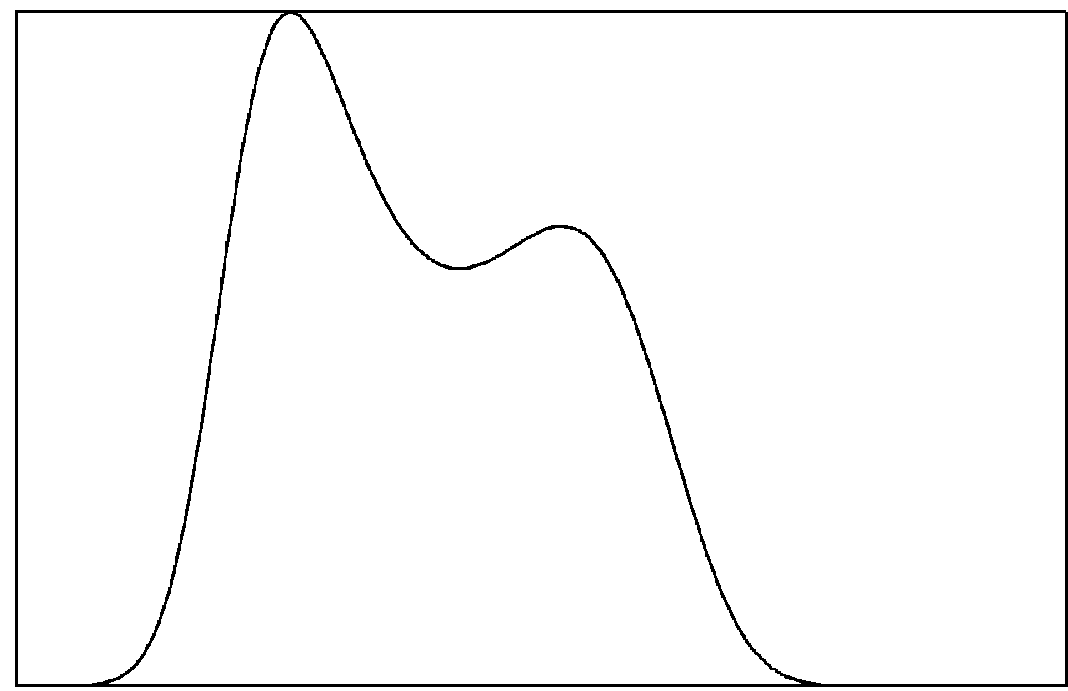

Figure 11: Optimal filter density at 4.12

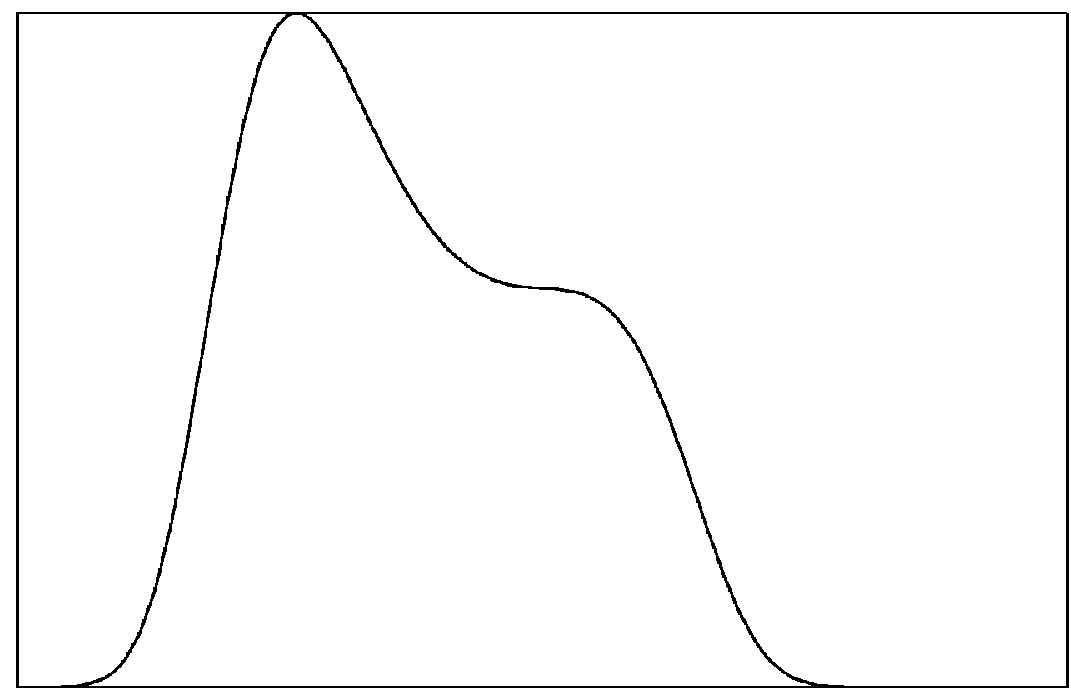

Figure 12: Projection filter density at 4.12 


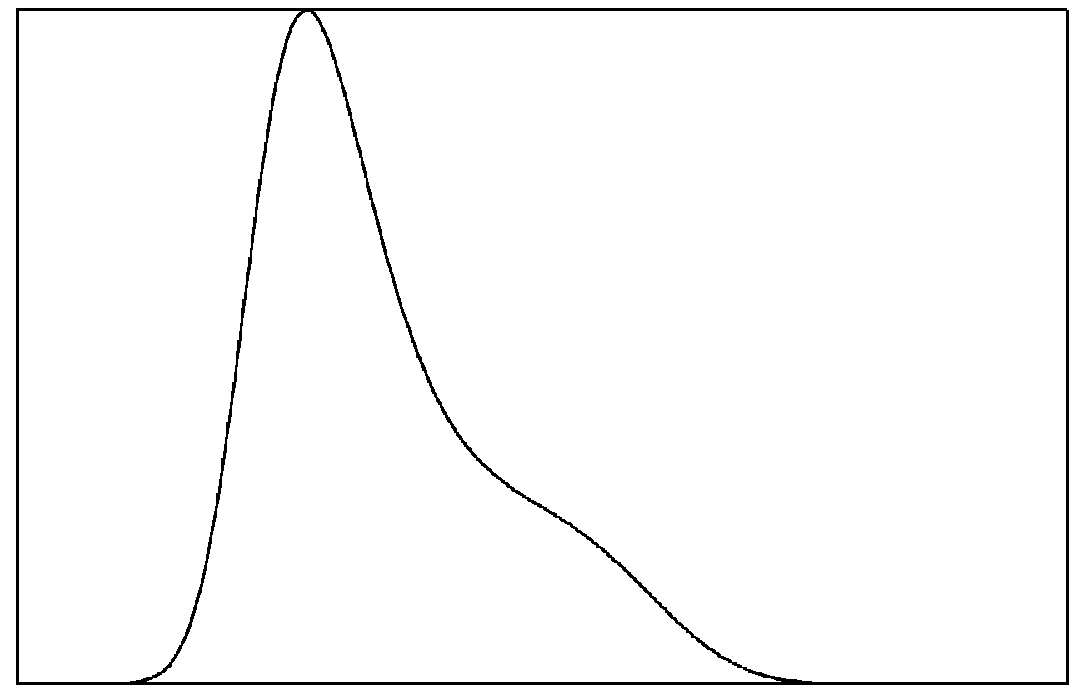

Figure 13: Optimal filter density at 9.54

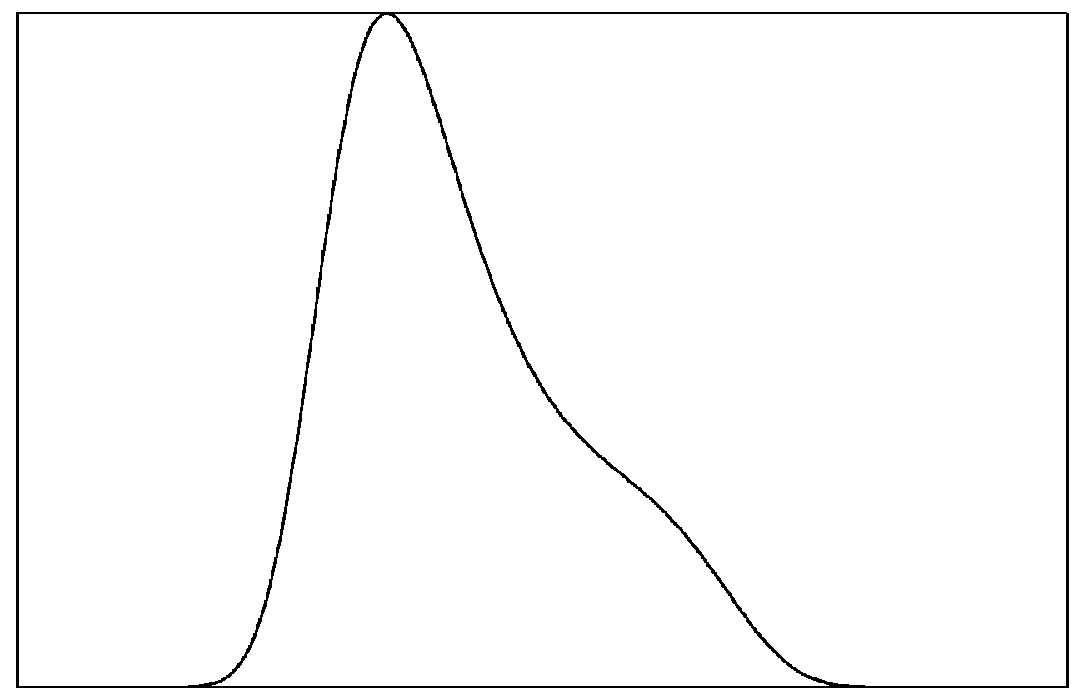

Figure 14: Projection filter density at 9.54 


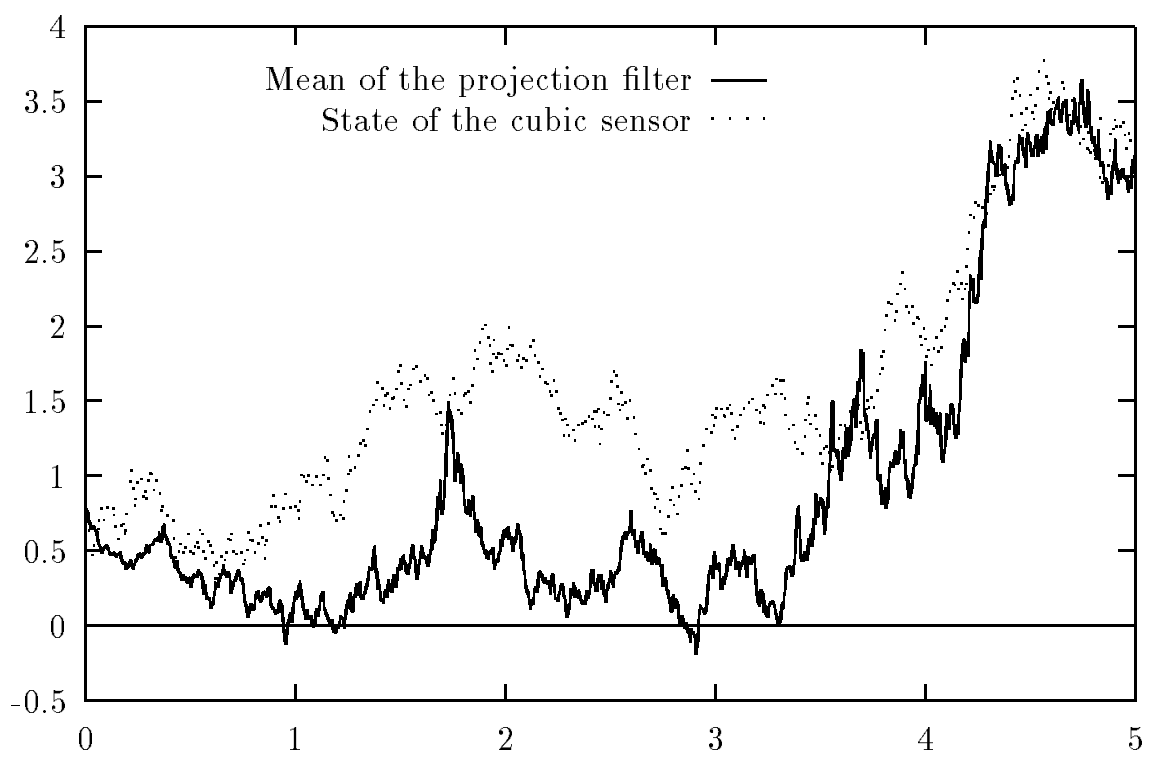

Figure 15: True state and mean from the projection filter between 0 and 5

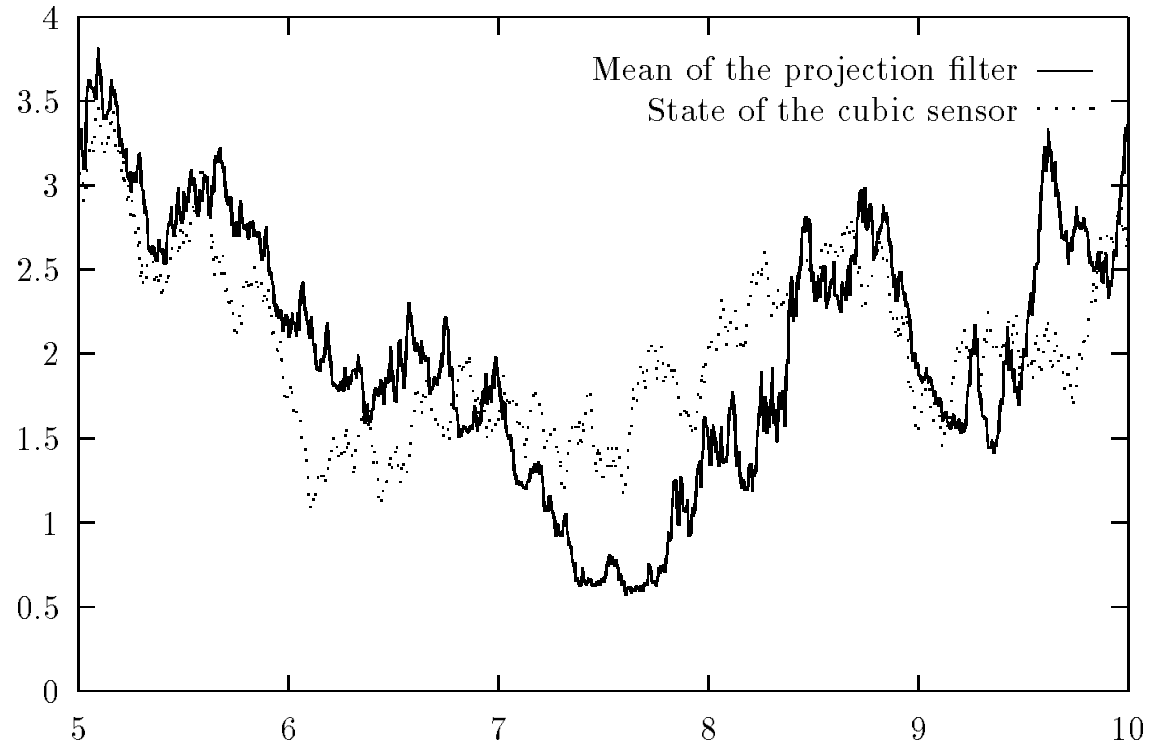

Figure 16: True state and mean from the projection filter between 5 and 10 


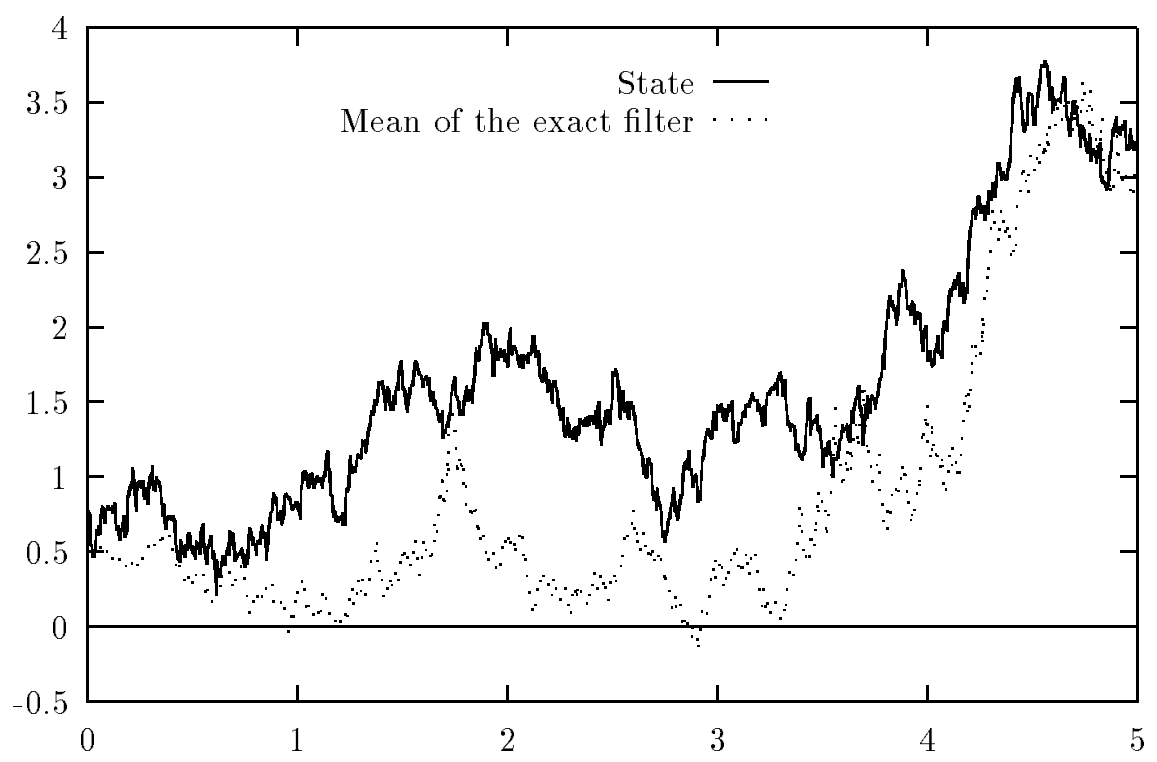

Figure 17: True state and mean from the optimal filter between 0 and 5

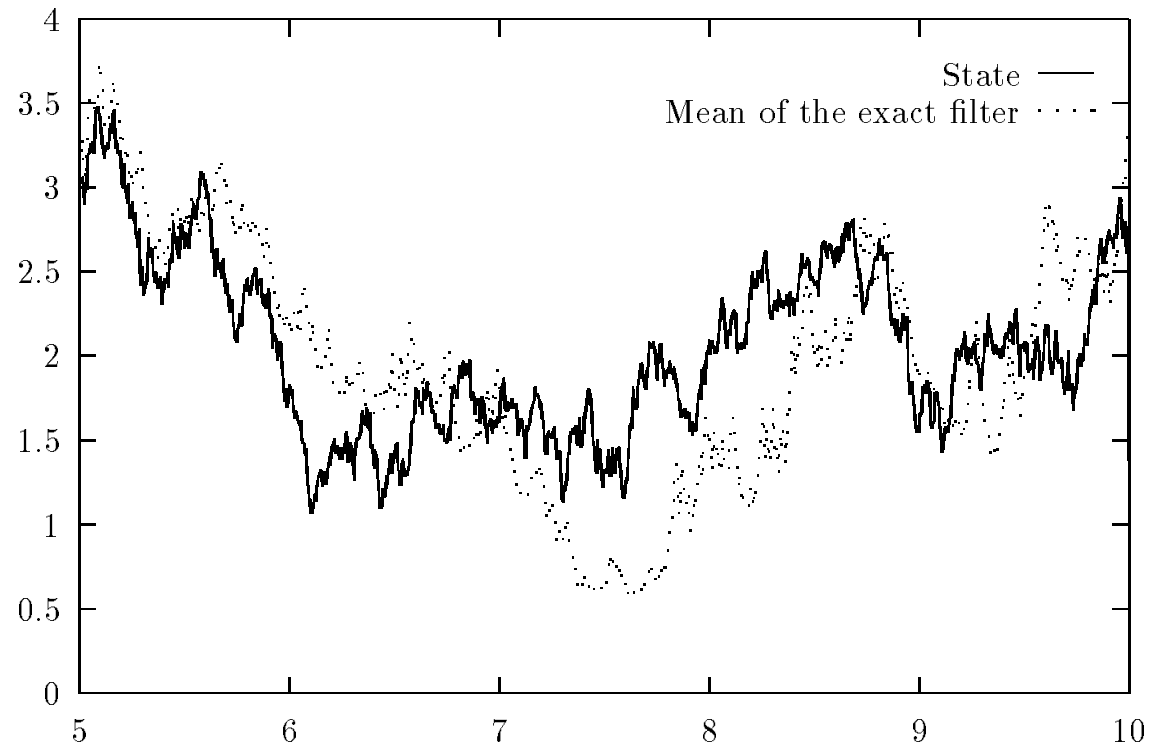

Figure 18: True state and mean from the optimal filter between 5 and 10 


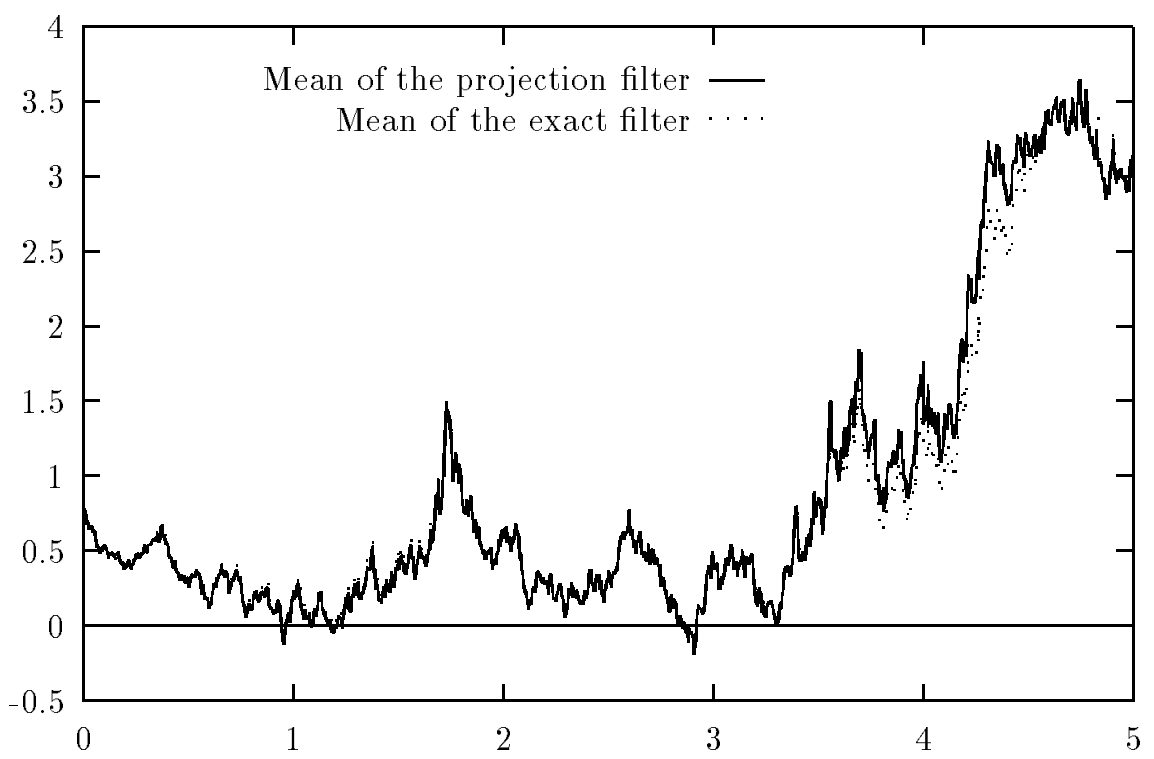

Figure 19: Mean from the projection filter and from the optimal filter between 0 and 5

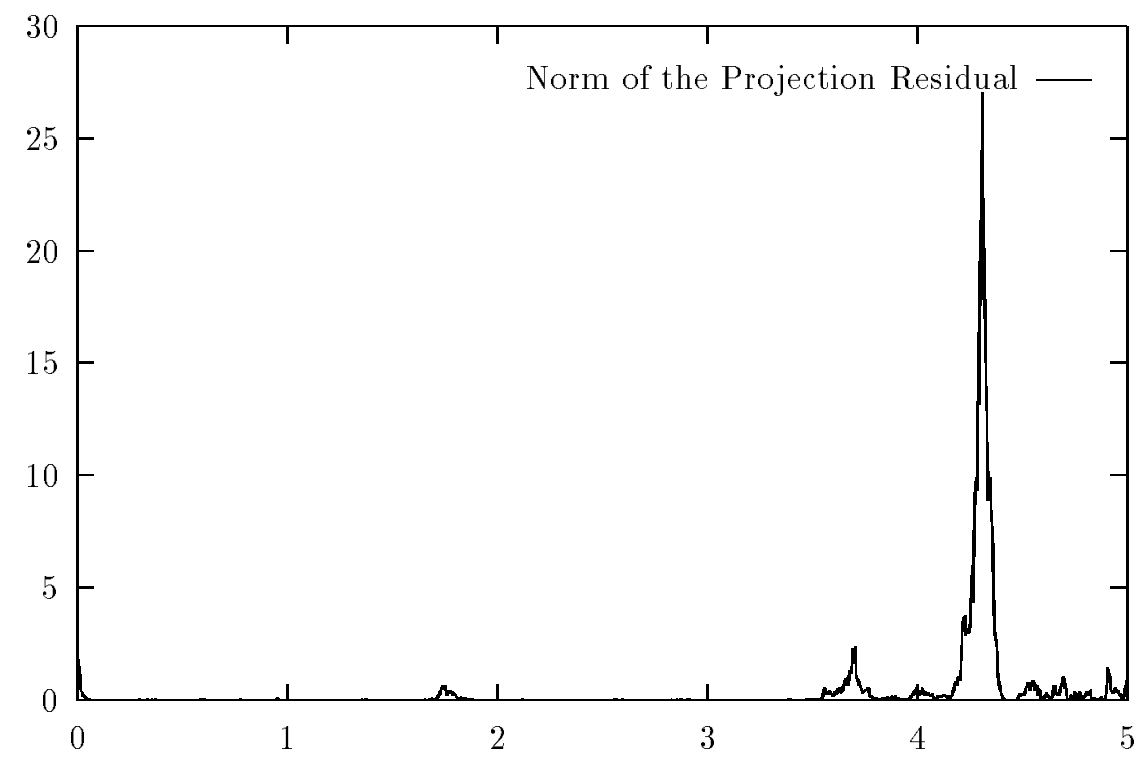

Figure 20: Projection residual between 0 and 5 


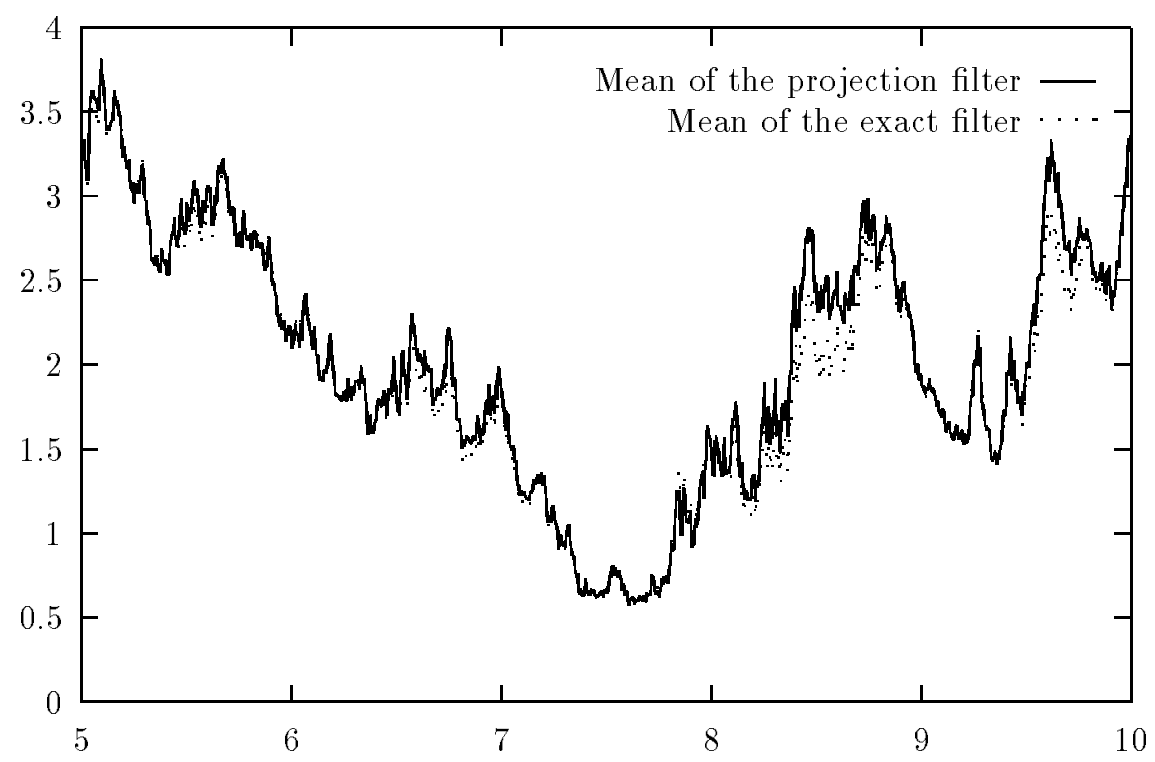

Figure 21: Mean from the projection filter and from the optimal filter between 5 and 10

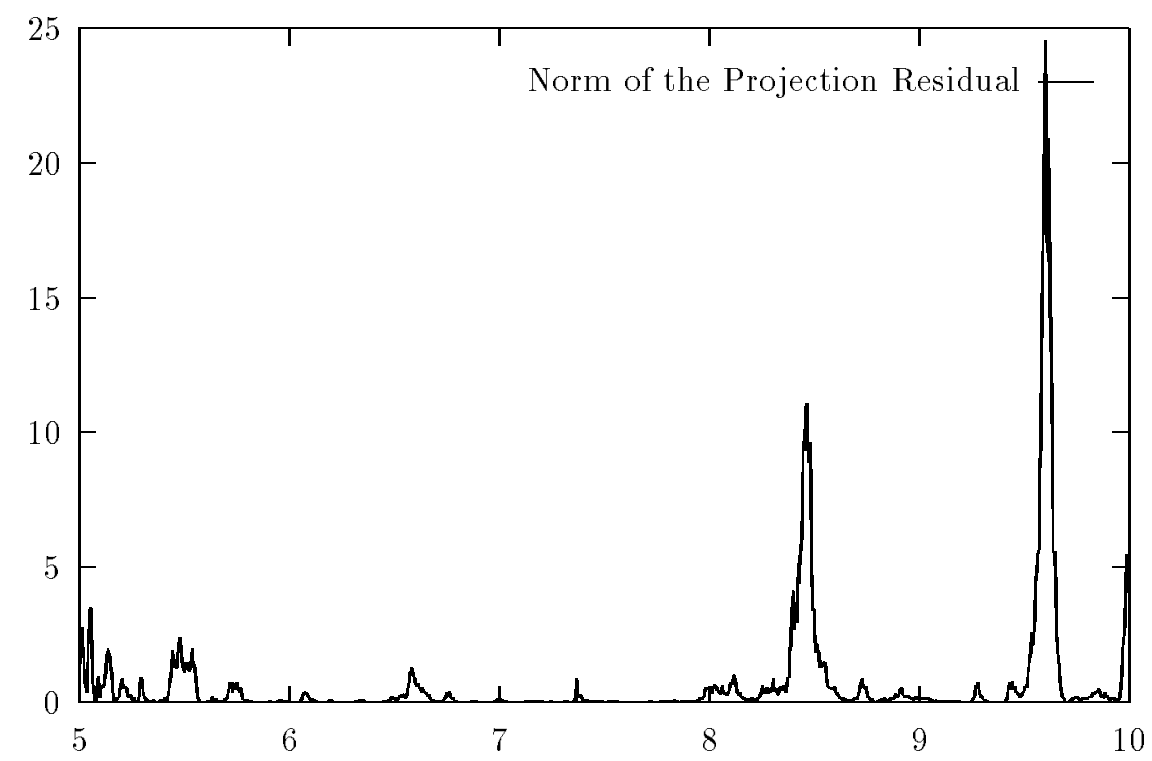

Figure 22: Projection residual between 5 and 10 


\section{Conclusion}

In this paper, we have introduced a new and systematic way of designing approximate finite-dimensional filters.

One major issue left is the choice of the exponential family $S$. A first answer has been given in Section 6, but this does not completely solve the problem : with the choice of the

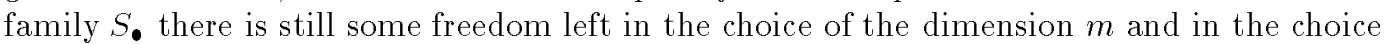
of the remaining functions $\left\{c_{s+1}, \cdots, c_{m}\right\}$, which could be used to reduce the total residual norm $r_{t}^{*}=r_{t}^{\bullet}$.

This freedom could also be used to design an adaptive scheme for the choice of the exponential family $S$.

It would also be useful to obtain for all $t \geq 0$ an estimate of the distance between the optimal filter density $p_{t}$ and the projection filter density $p_{t}^{\pi}$, in terms of the total residual norm history $\left\{r_{s}^{*}, 0 \leq s \leq t\right\}$.

Finally, we would like to define projection filters for discrete time systems, and relate this problem with the work of Kulhavý [13], [14]. Another motivation for this study will be to obtain efficient numerical schemes for the solution of the stochastic differential equation satisfied by the projection filter parameters, i.e. equation (19) for a general family $S$, or equation (30) for the family $S_{\text {. }}$.

Each of these problems requires further investigation, and we hope to address all of them in a subsequent work.

\section{References}

[1] Shun-ichi AMARI. Differential-Geometrical Methods in Statistics. Volume 28 of Lecture Notes in Statistics, Springer Verlag, Berlin, 1985.

[2] D. BRIGO. On the nice behaviour of the Gaussian projection filter with small observation noise. In Proceedings of the 3rd European Control Conference, Roma 1995, 1995.

[3] M.H.A. DAVIS and S.I. MARCUS. An introduction to nonlinear filtering. In M. Hazewinkel and J.C. Willems, editors, Stochastic Systems: the Mathematics of Filtering and Identification and Applications, Les Arcs 1980, D. Reidel, Dordrecht, 1981.

[4] P. FLORCHINGER and F. LE GLAND. Time discretization of the Zakai equation for diffusion processes observed in correlated noise. Stochastics and Stochastics Reports, 35(4):233-256, 1991.

[5] M. FUJISAKI, G. KALLIANPUR, and H. KUNITA. Stochastic differential equations for the non-linear filtering problem. Osaka Journal of Mathematics, 9(1):19-40, 1972.

[6] B. HANZON. A differential-geometric approach to approximate nonlinear filtering. In C.T.J. Dodson, editor, Geometrization of Statistical Theory, pages 219-223, ULMD Publications, University of Lancaster, 1987.

$\operatorname{RR} \mathrm{n}^{\circ} 2598$ 
[7] B. HANZON. Identifiability, Recursive Identification, and Spaces of Linear Dynamical Systems. CWI Tracts 63-64, CWI, Amsterdam, 1989.

[8] B. HANZON and R. HUT. New results on the projection filter. In Proceedings of the 1st European Control Conference, Grenoble 1991, pages 623-628, 1991.

[9] M. HAZEWINKEL, S.I. MARCUS, and H.J. SUSSMANN. Nonexistence of finite dimensional filters for conditional statistics of the cubic sensor problem. Systems $\&$ Control Letters, 3(6):331-340, 1983.

[10] A.H. JAZWINSKI. Stochastic Processes and Filtering Theory. Volume 64 of Mathematics in Science and Engineering, Academic Press, New York, 1970.

[11] R.Z. KHASMINSKII. Stochastic Stability of Differential Equations. Sijthoff and Noordhoff, Alphen aan den Rijn, 1980.

[12] P.E. KLOEDEN and E. PLATEN. Numerical Solution of Stochastic Differential Equations. Volume 23 of Applications of Mathematics, Springer Verlag, New York, 1992.

[13] R. KULHAVÝ. Recursive nonlinear estimation : a geometric approach. Automatica, 26(3):545-555, 1990.

[14] R. KULHAVÝ. Recursive nonlinear estimation : geometry of a space of posterior densities. Automatica, 28(2):313-323, 1992.

[15] S.I. MARCUS. Algebraic and geometric methods in nonlinear filtering. SIAM Journal on Control and Optimization, 22(6):817-844, 1984.

[16] P.S. MAYBECK. Stochastic Models, Estimation, and Control. Volume D. Volume 141-2 of Mathematics in Science and Engineering, Academic Press, New York, 1979.

[17] J. PICARD. Efficiency of the extended Kalman filter for nonlinear systems with small noise. SIAM Journal on Applied Mathematics, 51(3):843-885, June 1991.

[18] J. PICARD. Estimation of the quadratic variation of nearly observed semimartingales with application to filtering. SIAM Journal on Control and Optimization, 31(2):494517, March 1993.

[19] J. PICARD. Nonlinear filtering of one-dimensional diffusions in the case of a high signal-to-noise ratio. SIAM Journal on Applied Mathematics, 46(6):1098-1125, December 1986. 


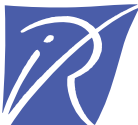

Unité de recherche INRIA Lorraine, Technopôle de Nancy-Brabois, Campus scientifique, 615 rue du Jardin Botanique, BP 101, 54600 VILLERS LES NANCY

Unité de recherche INRIA Rennes, Irisa, Campus universitaire de Beaulieu, 35042 RENNES Cedex

Unité de recherche INRIA Rhône-Alpes, 46 avenue Félix Viallet, 38031 GRENOBLE Cedex 1

Unité de recherche INRIA Rocquencourt, Domaine de Voluceau, Rocquencourt, BP 105, 78153 LE CHESNAY Cedex

Unité de recherche INRIA Sophia-Antipolis, 2004 route des Lucioles, BP 93, 06902 SOPHIA-ANTIPOLIS Cedex

Éditeur

INRIA, Domaine de Voluceau, Rocquencourt, BP 105, 78153 LE CHESNAY Cedex (France)

ISSN 0249-6399 